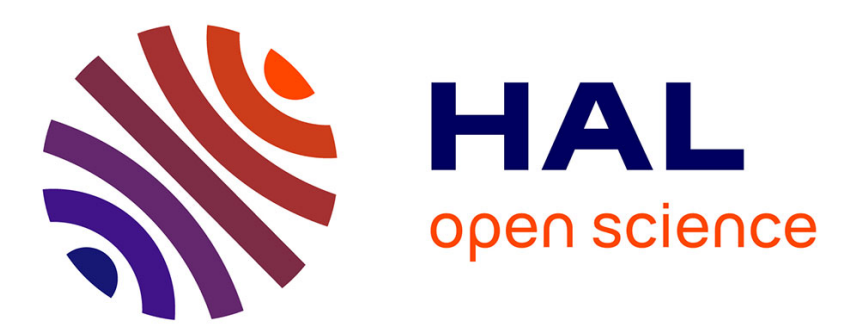

\title{
Synchronization of acquisition devices in neuroimaging: An application using co-registration of eye movements and electroencephalography
}

Gelu Ionescu, Aline Frey, Nathalie Guyader, Emmanuelle Kristensen, Anton Andreev, Anne Guérin-Dugué

\section{To cite this version:}

Gelu Ionescu, Aline Frey, Nathalie Guyader, Emmanuelle Kristensen, Anton Andreev, et al.. Synchronization of acquisition devices in neuroimaging: An application using co-registration of eye movements and electroencephalography. Behavior Research Methods, 2021, 10.3758/s13428-021-01756-6 . hal03484802

\author{
HAL Id: hal-03484802 \\ https://hal.science/hal-03484802
}

Submitted on 11 Feb 2022

HAL is a multi-disciplinary open access archive for the deposit and dissemination of scientific research documents, whether they are published or not. The documents may come from teaching and research institutions in France or abroad, or from public or private research centers.
L'archive ouverte pluridisciplinaire HAL, est destinée au dépôt et à la diffusion de documents scientifiques de niveau recherche, publiés ou non, émanant des établissements d'enseignement et de recherche français ou étrangers, des laboratoires publics ou privés. 
Synchronization of acquisition devices in neuroimaging:

An application using co-registration of eye movements and electroencephalography

Gelu Ionescu $^{\dagger}$, Aline Frey ${ }^{2}$, Nathalie Guyader ${ }^{1}$, Emmanuelle Kristensen $^{1}$, Anton Andreev ${ }^{1}, \&$ Anne Guerin-Dugué ${ }^{1}$

${ }^{1}$ Univ. Grenoble Alpes, CNRS, Grenoble INP*, GIPSA-lab, 38000 Grenoble, France

* Institute of Engineering Univ. Grenoble Alpes

${ }^{2}$ Laboratoire de Neurosciences Cognitives, UMR 7291, CNRS - INSPE d'Aix-Marseille Université, $13 \quad$ France

Corresponding author:

Anne Guérin-Dugué, GIPSA-lab,

11 rue des Mathématiques,

21 Grenoble Campus BP46,

22 F-38402 Saint Martin d'Hères Cedex

23 E-mail : anne.guerin@gipsa-lab.grenoble-inp.fr

$24 \quad$ Tel: +33(0)4 76574790 
Synchronization of acquisition devices in neuroimaging:

An application using co-registration of eye movements and electroencephalography Abstract

Interest in applications for the simultaneous acquisition of data from different devices is growing. In

28 neuroscience for example, co-registration complements and overcomes some of the shortcomings of

29 individual methods. However, precise synchronization of the different data streams involved is required

30 before joint data analysis. Our article presents and evaluates a synchronization method which maximizes

31 the alignment of information across time.

32 Synchronization through common triggers is widely used in all existing methods, because it is very

33 simple and effective. However, this solution has been found to fail in certain practical situations, namely

34 for the spurious detection of triggers and/or when the timestamps of triggers sampled by each acquisition

35 device are not jointly distributed linearly for the entire duration of an experiment. We propose two

36 additional mechanisms, the "Longest Common Subsequence" algorithm and a piecewise linear regression,

37 in order to overcome the limitations of the classical method of synchronizing common triggers.

38 The proposed synchronization method was evaluated using both real and artificial data. Co-

39 registrations of electroencephalographic signals (EEG) and eye movements were used for real data. We

40 compared the effectiveness of our method to another open source method implemented using EYE-EEG

41 toolbox. Overall, we show that our method, implemented in $\mathrm{C}++$ as a DOS application, is very fast, robust 42 and fully automatic.

\section{Keywords}

45 Co-registration, Synchronization, Clock drift, Drift correction, Electroencephalography, Eye 46 movements. 
Synchronization of acquisition devices in neuroimaging:

An application using co-registration of eye movements and electroencephalography

\section{Synchronization of acquisition devices in neuroimaging:}

An application using co-registration of eye movements and electroencephalography

\section{Introduction}

In neuroimaging, simultaneous recordings are a powerful way of investigating brain activity. All

53 experimental modalities have their own advantages and limitations. They also have a certain degree of

54 complementarity, mainly in the temporal domain (e.g. for EEG + eye movements, and in both the temporal

55 and spatial domains (e.g., for EEG + fMRI), which has motivated intense efforts towards their combination

56 (Jorge et al., 2015; Rosenkranz \& Lemieux, 2010). Two main approaches coexist. The first approach - data

57 integration - uses one modality to improve another, for example, when the high spatial resolution of

58 functional Magnetic Resonance Imaging (fMRI) is used to constrain electroencephalography (EEG) inverse

59 solutions for source localization (Liu, Ding \& He, 2006). The second approach - data fusion - requires

60 synchronous acquisition, and refers to the idea of using both modalities in a combined joint analysis, for

61 example for the simultaneous recording of EEG and near-infrared spectroscopy ( Shin, von Lühmann, Kim,

62 Mehnert, Hwang \& Müller, 2018), or for EEG and eye movements ( Nikolaev, Meghanathan \& van

63 Leeuwen, 2016).

Ideally, one single data acquisition system would record all necessary data types, inherently time-

65 synchronizing all measurements. In many cases however, multiple data acquisition systems are involved,

66 and efficient data synchronization is needed to harmonize data continuously over time. Off-line

67 synchronization methods rely on matching information from two or more simultaneous measurements

68 (Hoogeboom, 2003). As a result of the synchronization, all data are sampled in the same time referential

69 and share the same events. 
Synchronization of acquisition devices in neuroimaging:

An application using co-registration of eye movements and electroencephalography

In this context of data fusion, we propose a solution for the synchronization of data from different acquisition devices, and we compare it to a currently existing solution in terms of limitations and

73 advantages. We illustrated our method by testing it on real research data obtained during the co-registration

74 of EEG signals and eye movements. In this type of experimental setup, the EEG signal is segmented based

75 on eye movement events, by using the temporal and spatial positions of specific eye events (e.g., fixations

76 or saccades) as markers for brain signals. These EEG analyses, triggerred by eye events, are called Eye

77 Fixation or Saccade Related Potentials (EFRP / ESRP), and can be analyzed using efficient methods of

78 estimation (Kristensen, Guerin-Dugué \& Rivet, 2017; Kristensen, Rivet \& Guérin-Dugué, 2017; Ehinger \&

79 Dimigen, 2019). This co-registration technique is an effective way of delving into cognitive processes

80 (Frey, Lemaire, Vercueil \& Guérin-Dugué, 2018). It also allows for more ecological experimental

81 protocols, which are not constrained by "external" markers, such as the onset of a word or image, and in

82 which participants can perform fixations at their own pace (Dimigen, Sommer, Hohlfeld, Jacobs, \& Kliegl,

83 2011; Körner, Braunstein, Stangl, Schlögl, Neuper \& Ischebeck, 2014; Van Humbeeck, Meghanathan,

84 Wagemans, van Leeuwen \& Nikolaev, 2018).

85 It is being used increasingly to study attention, memory encoding, visual scene processing, reading, and 86 responses to emotional visual information (for reviews, cf. for example (Dimigen, et al., 2011; Nikolaev, 87 Pannasch, Ito \& Belopolsky, 2014; Nikolaev et al., 2016). This type of joint acquisition therefore has a 88 strong added value for the understanding of the time course of neural activity during cognitive tasks 89 requiring a high degree of accuracy in the synchronization of timings.

90 Where systems of acquisition are concerned, suppliers of both eye-trackers and EEG systems have 91 recently proposed additional modules which permit the importation and synchronization of co-registered 92 data streams. The EEG analysis software 'BrainVision Analyzer' (Brain Products GmbH) proposed a first 
Synchronization of acquisition devices in neuroimaging:

An application using co-registration of eye movements and electroencephalography

93 version of the "Add Channels" module, supporting four types of eye-tracker data: Tobii (TobiiStudio223

94 and TobiiStudio301), ASL (ASLEyeTrac6 and ASLResults243), SMI (SMIBeGaze300) and SR Eye Link

951000 Plus. The whole process is based on common triggers, found by the module, for the drift correction.

96 Gaze positions are then resampled and added to EEG channels (Brain Products Press Release, 2013 ${ }^{1}$ ). The

97 Tobii system proposed the Pro Lab software which uses Transistor-Transistor Logic (TTL) technology to

98 send events from the parallel port to the other devices for synchronization ${ }^{2}$. Finally, iMotions combined

99 Eye Tracking glasses with a wireless EEG device. In addition to these corporate solutions, which need to be

100 purchased separately, the open source EEGLab environment (Delorme \& Makeig, 2004) has developed a

101 plug-in for the joint analysis of EEG signals and eye-tracker data (EYE-EEG ${ }^{3}$, Dimigen et al., 2011). This

102 plug-in also resolves the synchronization issue between these devices thanks to common triggers in the two

103 data flows.

104 Thus, although several solutions exist, no consensus on an effective synchronization process seems

105 to have emerged, and in general, each study attempts to find its own solution. However, it appears that in

106 most cases, common triggers are sent as TTL pulses, on a parallel port, typically at the beginning of each

107 trial (Dimigen et al., 2011; Guérin-Dugué, Roy, Kristensen, Rivet, Vercueil, \& Tcherkassof, 2018; Van

108 Humbeeck et al., 2018). Moreover, due to several potential problems, which will be discussed in this

109 article, this synchronization procedure requires realignment precautions, and it is important to ensure its

110 effectiveness. As mentioned above, the point of these co-registration methods is often to obtain good

\footnotetext{
${ }^{1} \mathrm{https}: / /$ www.brainproducts.com/analyzer203.php?tab $=4$

${ }^{2}$ https://www.tobiipro.com/learn-and-support/learn/steps-in-an-eye-tracking-study/design/solutions-for-co-registration-of-eyetracking-and-other-biometric-measures/

${ }^{3}$ http://www2.hu-berlin.de/eyetracking-eeg/
} 
Synchronization of acquisition devices in neuroimaging:

An application using co-registration of eye movements and electroencephalography

111 temporal resolution and a few milliseconds of lag can be very damaging to the analysis and understanding 112 of the results.

113 As an example, Kamienkowski, Ison, Quiroga, and Sigman (2012)evaluated the synchronization procedure

114 by comparing the onset of saccades detected by electro-oculography (EOG) channels to the onset of

115 saccades detected by the Engbert and Kliegl (2003) algorithm in the eye-tracking recording, which showed

116 lags between saccade onsets of under $20 \mathrm{~ms}$ in all trials. In the latest version of the EYE-EEG ${ }^{3}$ toolbox,

117 synchronization accuracy can be checked by cross-correlation of eye positions and EOG channels for both

118 horizontal and vertical movements. For successful synchronization, the lag that maximizes the cross-

119 correlation must be close to zero.

120 In the following sections, we describe the framework for synchronizing independent acquisition devices

121 and the practical difficulties that can be encountered. We implemented the proposed method in a software

122 named "GazeEEGSynchro", written in $\mathrm{C}++$ and implemented as a DOS application, that is provided and

123 available in the Zenodo repository (https://doi.org/10.5281/zenodo.5554071) with examples on real data.

124 We thus describe our proposed approach, validated with simulated and real data -acquired by our research

125 team and by a team from another laboratory-, in order to evaluate its effectiveness, and we compare it with 126 the EYE-EEG toolbox.

\section{Trigger-based synchronization}

When processing recorded signals off-line, it is essential to readjust these signals into the same time

131 referential because of the inaccuracy of the crystal-based clock of each individual device (clock drift issue).

132 Trigger-based synchronization is the simplest and most commonly used solution for the synchronization of 133 devices working at the same or at different sampling rates. In this section, we begin by describing the clock 
Synchronization of acquisition devices in neuroimaging:

An application using co-registration of eye movements and electroencephalography

134 drift issue between the oscillators of each acquisition device, and the synchronization principle usually

135 employed to resolve this.

137 Clock drift issue

In an acquisition system with several acquisition devices, each device's clock is controlled by its

139 own crystal oscillator. Even when two quartz crystals come from the same batch, oscillators can exhibit a

140 variety of instabilities related to aging, frequency, changes in temperature, power supply inaccuracy, and

141 wire interconnection lengths. The phenomenon whereby a clock does not run at the same nominal speed as

142 an ideal clock is called clock drift (Pak, 2017). In practice, quartz crystals are manufactured for frequencies

143 ranging from a few tens of $\mathrm{kHz}$ to tens of $\mathrm{MHz}$, and are often designed around standard frequencies ${ }^{4}$ such

144 as $3.579545 \mathrm{MHz}$ adopted by the National Television System Committee, $10 \mathrm{MHz}$ for low-power

145 microcontrollers, $33.33 \mathrm{MHz}$ or $40 \mathrm{MHz}$ for computers. A programmable frequency divider is also

146 necessary, because in acquisition systems the desired sampling frequency should be programmable in a

147 range of tens of $\mathrm{Hz}$ to several tens of $\mathrm{kHz}$. Therefore, the clock drift of the operating frequency also has an

148 impact on these frequency dividers. Consequently, efficient synchronization is needed to compensate for

149 the relative differences in timing generated by acquisition systems affected by different degrees of clock

150 drift.

\footnotetext{
${ }^{4}$ https://en.wikipedia.org/wiki/Crystal_oscillator_frequencies
} 
Synchronization of acquisition devices in neuroimaging:

An application using co-registration of eye movements and electroencephalography

152

153

154 simple and generic experimental design which involves two acquisition devices and a master computer, as 155 depicted in Figure 1.

\section{Principle of trigger-based synchronization}

We describe the trigger-based synchronization aimed at solving the clock drift issue using a very

The STIMULATION PC (master) is used to present external stimuli (visual, auditory or both) to the participant, to record if necessary their response, and send triggers through a logical bus (TTLB). The triggers are used to tag the different stages of the experiment (start, end, visual display, sound emission, etc.), and they are received instantly by all acquisition devices. Two acquisition devices (ACQD1 and ACQD2), controlled by two independent PCs (HOST PC1 and HOST PC2), complete this set-up. The STIMULATION PC communicates with the two HOST PCs through two control buses (CB1 respectively CB2; i.e. Ethernet, USB, etc.). Likewise, the two HOST PCs communicate with the acquisition devices through two control/data buses (CDB1 and CDB2, i.e. Ethernet, USB or PCI). These buses are used to control the acquisition devices and to transfer the acquired data (digitized analog signals and TTL triggers). In this way, data are continuously stored in two different files on the corresponding HOST PCs.

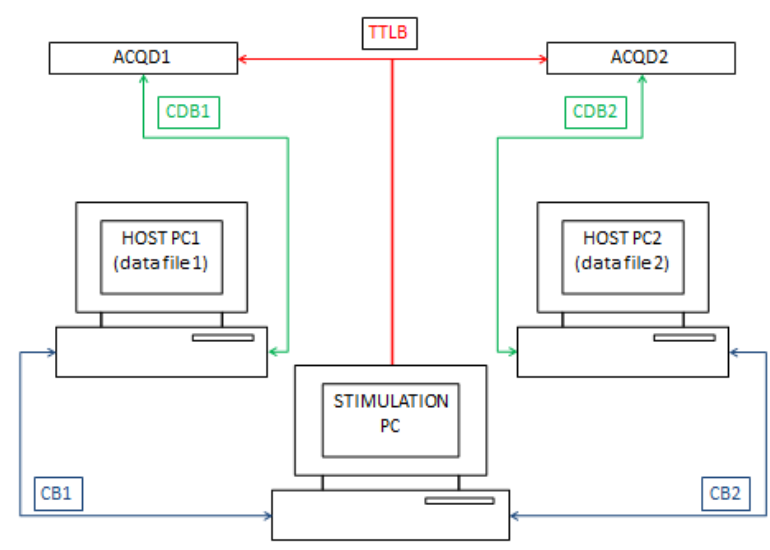

168 Figure 1. Example of an acquisition system with two acquisition devices: ACQD1 and ACQD2; three PCs: 169 STIMULATION PC (master), HOST PC1 and HOST PC2 (acquisition devices); two bidirectional 
Synchronization of acquisition devices in neuroimaging:

An application using co-registration of eye movements and electroencephalography

170

171

172

173

174

175

176

177

178

179

180

181

182

183

184

185

186

187

188

189

190

191

192

control/data buses CDB1 and CDB2 (communication between each host PC and the acquisition device); two bidirectional control buses CB1 and CB2 (communication between the STIMULATION PC and the two HOST PCs); a logical bus TTLB (trigger support).

A synchronization procedure requires at least two common triggers to be registered by both acquisition devices. One trigger is sent just after the beginning of the experiment ("start trigger") and another trigger is sent just before the end of the experiment ("end trigger"), and both are recorded by acquisition devices ACQD1 and ACQD2. A time reference is needed for synchronization purposes. This time reference can be provided either by one of the acquisition devices or by the master computer. Once the reference device has been selected, the other devices are called "secondary devices". The timestamps of the triggers of each secondary device are then linearly interpolated between the "start trigger" and the "end trigger" to align with the timestamps of the triggers from the reference device.

If we consider that $N_{T}$ triggers were sent throughout the whole experiment, the formula applied for the transformation of the sample index of the trigger is:

$$
s_{j 1}^{(k)}=\left(s_{j}^{(k)}-s_{j}^{(1)}\right) \frac{s_{1}^{\left(N_{T}\right)}-s_{1}^{(1)}}{s_{j}^{\left(N_{T}\right)}-s_{j}^{(1)}}+s_{1}^{(1)}
$$

where $\left.\in\left\{2,3, \ldots, N_{D}\right\}\right\}$ is the index of the secondary acquisition devices for a set-up with $N_{D}$ devices (index 1 is reserved for the reference device), and $k \in\left\{1,2, \ldots, N_{T}\right\}$ is the trigger index with $N_{T}$ the number of common triggers detected by the acquisition devices. Then, for the reference device and the $j^{\text {th }}$ device, $s_{1}^{(k)}$ and $s_{j}^{(k)}$ are the trigger indexes of the $k^{t h}$ trigger in each respective data acquisition flow expressed in number of samples. In this context, $s_{j 1}^{(k)}$ represents the new position of the $k^{t h}$ trigger from the $j^{\text {th }}$ device after its alignment with $s_{1}^{(k)}$ in the data acquisition flow of the reference device (index 1 ). In this context, the alignment error $\varepsilon_{s}^{(k)}$ that affects the $k^{\text {th }}$ trigger transformation, expressed in number of samples (subscript " $s ")$ can be computed as: 
Synchronization of acquisition devices in neuroimaging:

An application using co-registration of eye movements and electroencephalography

$$
\varepsilon_{s}^{(k)}=\operatorname{round}\left(s_{1}^{(k)}-s_{j 1}^{(k)}\right)
$$

194 The errors $\left\{\varepsilon_{s}^{(k)}, k=2, \ldots, N_{T}\right\}$ are theoretically bound by the ratio between the sampling periods of the 195 acquisition devices. In Figure $2 A$, the sampling mechanism is schematized for an extreme case: an 196 asynchronous trigger $T$ (top line) appears slightly before the sampling time of the reference device (middle 197 line), and slightly after the sampling time of a secondary device (bottom line). Thus, the maximum 198 uncertainty, expressed in time (subscript " $t^{\prime \prime)}$, is $\varepsilon_{t}= \pm \max \left(T_{i}\right)$ where $T_{i}$ is the sampling period of the $199 i^{t h}$ acquisition device. By introducing $R_{T}$ as the sampling ratio so that $R_{T}=\max \left(T_{i}\right) / \min \left(T_{i}\right)$, the 200 maximum uncertainty can be rewritten as $\varepsilon_{t}= \pm R_{T} \cdot \min \left(T_{i}\right)$. For the faster device, the same error, 201 expressed in terms of number of samples $\left(\varepsilon_{S}\right)$ can then be written as $\varepsilon_{S} \in\left[-R_{T}, R_{T}\right]$.

To illustrate the performance of this synchronization procedure, we simulated ten thousand 204 experiments of about one hour using two independent acquisition devices. Each device had its own time referential, its own nominal sampling frequency and its own random clock drift. With a 1\%o clock drift

206 value being considered as usual, a random clock drift chosen in the interval $\left[-\frac{F}{1000}, \frac{F}{1000}\right]$ was added to the 207 nominal sampling rate $F$ of the corresponding acquisition device. Two situations were simulated. In the 208 first one, the reference device had a sampling frequency ten times greater than the secondary device $(F 1=$ $\left.20910 \mathrm{kHz}, \mathrm{F2}=1 \mathrm{kHz}, R_{T}=10\right)$. In the second case, both devices had the same sampling frequency $210\left(F 1=1 \mathrm{kHz}, F 2=1 \mathrm{kHz}, R_{T}=1\right)$. The nominal sampling rates were affected by random clock drifts, 211 as explained previously. For each simulation, the asynchronous triggers were added into recorded signals 212 from both devices by an independent uniform random generator. On average there were $N_{T}=3273$ 213 asynchronous triggers per experiment. The transmission delay towards the acquisition devices was not 
Synchronization of acquisition devices in neuroimaging:

An application using co-registration of eye movements and electroencephalography

considered. The only uncertainty was, therefore, the date at which trigger signals were sampled according

215 to the sampling clock of each acquisition device (Figure $2 A$ ).
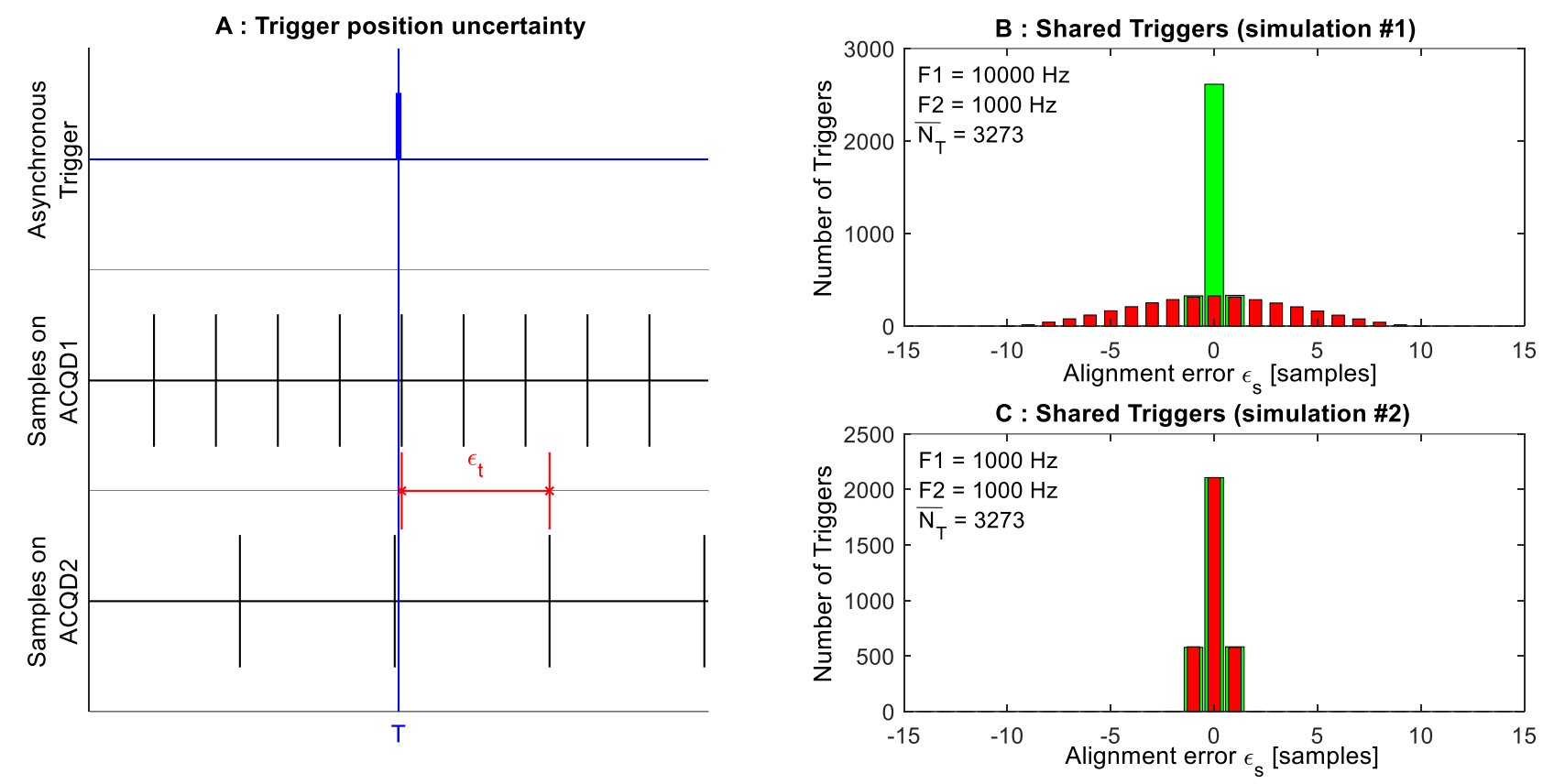

Figure 2. Trigger-based synchronization. A. Trigger uncertainty overview in an extreme case when the trigger $\mathrm{T}$ arrives just before and just after the sampling fronts. The vertical black segments represent the samples acquired by the ACQD1 and ACQD2 devices working at different sampling rates. B. Averaged histogram of alignment errors in samples $\left(\varepsilon_{S}\right)$ with $R_{T}=10$. C. Averaged histogram of alignment errors in samples $\left(\varepsilon_{S}\right)$ with $R_{T}=1$, . The red histogram illustrates errors from the fastest acquisition device compared to the slowest one, while the green histogram represents the reciprocal situation. Keys: $F 1$ - ACQD1 sampling rate -; F2 - ACQD2 sampling rate -; $\overline{N_{T}}$ - average number of triggers detected by ACQD1 and ACQD2 -.

The range of the synchronization errors must be $\varepsilon_{s} \in[-10,10]$ in the first case, and $\varepsilon_{s} \in[-1,1]$ in

228 the second case. These expected results are confirmed by the histograms of alignment errors $\left(\varepsilon_{s}\right)$, averaged

229 across all simulated experiments. These histograms are shown in Figure $2 B\left(R_{T}=10\right)$ and in Figure $2 C$

$230\left(R_{T}=1\right)$. The observed range is slightly larger when round-off errors are taken into consideration. 
Synchronization of acquisition devices in neuroimaging:

An application using co-registration of eye movements and electroencephalography

233 after the last trigger is sent, so that the start and end of the acquisitions are unambiguously specified by

234 unique triggers; (2) all triggers must be sampled and shared by each acquisition device; and (3) the

235 acquisition must be continuous, without any interruption, so that drifts are distributed linearly throughout

236 the entire experiment.

237 In this ideal experimental situation, linear alignment of all triggers with the reference time is

238 effective. However, because of handling errors, transient recording failures, false trigger detections and

239 pauses during acquisition to cite some examples, an ideal situation is not always attained. Our procedure,

240 which resolves these difficulties, is presented in the next section.

Description of the proposed synchronization procedure

The synchronization procedure proposed in this article efficiently corrects the clock drift even when

244 the experimental situation is not ideal. The procedure allows correction when missing and/or spurious

245 triggers are observed ("Processing missing and spurious triggers" section), and when interruptions in

246 acquisition occur ("Processing Pause/Resume mode" section).

\section{Processing missing and spurious triggers}

The causes of missing or spurious triggers between different trigger streams are many and

249 multifactorial. Triggers identified in the experimental design and sent by the "Stimulation PC" (Figure 1)

250 but not recorded by all devices, are called "missing" triggers, as they are missing from at least one device.

251 Triggers recorded by at least one device but not sent by the "Stimulation PC" are called spurious triggers.

252 Configurations with missing triggers can be observed in a number of situations. If one device is switched

253 on too late, or switched off too early, the first common trigger or the last common trigger determining the

254 beginning or the end of acquisition is lost. This handling error can also result in a loss of data, when data 
Synchronization of acquisition devices in neuroimaging:

An application using co-registration of eye movements and electroencephalography

255 and triggers recorded on one acquisition device are not recorded on the others. Transient recording failure

256 can have the same consequence. For example, a "buffer overflow" can occur in one system while the others

257 continue recording. Configurations with spurious triggers can be observed in a number of situations. The

258 detection of triggers is based on the electronic device that samples the signals on the parallel bus, and on

259 the configuration mode of the parallel port. The name of a trigger is based on the decimal value

260 corresponding to these sampled binary digits. We observed that the occurrence of additional spurious

261 triggers depended on the electronic device used to sample and lock the logic levels on the parallel port. For

262 example, in the analyzed recordings (cf. below the "Validation on real data" section), three different EEG

263 systems were used. For two of these, no spurious triggers were observed, and for the other, up to $20 \%$ of

264 the total number of triggers observed were spurious (Table 1). The spurious triggers observed corresponded

265 to transient states on one sampling period before the expected trigger. Because the rise times and fall times

266 can differ, their occurrence depended on the up or down transitions of logic levels on the parallel bus from

267 one value (the previous trigger) to another (the subsequent trigger). Consequently, these spurious triggers

268 appear in one trigger flow for a given device but not in the other trigger flows and have to be ignored. The

269 proposed procedure resolves all of these issues.

270 String comparison is a central operation in a number of situations such as: in the comparison of two

271 DNA sequences or in gene identification searches in molecular biology; in spelling error correction

272 programs aimed at finding the dictionary entry which most resembles a given word; in the detection of

273 plagiarism, and in pattern recognition (Needleman \& Wunsch, 1970; Pearson \& Lipman, 1988; Altschul,

274 Gish, Myers \& Lipman, 1990). As mentioned previously, when trigger flows from different devices are

275 being compared, the alignment procedure may be deficient in situations featuring missing or spurious

276 triggers. Before beginning the synchronization procedure, the common triggers in both acquisition flows

277 must be matched specifically. This allows the triggers that mark the beginning and the end of acquisition to 
Synchronization of acquisition devices in neuroimaging:

An application using co-registration of eye movements and electroencephalography

278 be defined in a unique way. The "Longest Common Subsequence" (LCS) algorithm is proposed here as a

279 way of resolving this problem (see Bergroth, Hakonen \& Raita (2000) for a review). The LCS of two

280 sequences $\mathrm{X}$ and $\mathrm{Y}$ constitutes a common subsequence of maximal length, i.e. the maximum number of

281 identical symbols in both sequences when the common order in which these symbols occur in both

282 sequences is maintained. The traditional implementation of the LCS between two sequences X and Y is by

283 dynamic programming with a computation time proportional to the product of the lengths of the two

284 sequences. The output is the longest sub-sequence with common triggers between the two sequences; the

285 position of the common triggers may not be contiguous.

286 Figure 3 illustrates two trigger sequences, based on real acquisitions, used as inputs to the LCS

287 algorithm (a first sequence recorded by the reference device called the reference Trigger sequence, $R T$, and

288 a second sequence recorded by a secondary device called the secondary Trigger sequence, ST), and a

289 unique trigger sequence $(A L L)$ as output of the LCS algorithm. Each trigger is identified by a name which

290 is based on the value transmitted in 8 bits by the parallel port to the acquisition devices. The two input

291 sequences were recorded during a short real acquisition session with only 32 triggers in the RT sequence.

292 The algorithm, based only on the triggers' position in the sequence, allowed us to obtain the longest

293 subsequence of common triggers $(A L L)$. The first and the last of these common triggers, which mark the

294 beginning and the end of the synchronization procedure respectively, are of particular importance to the

295 subsequent application of drift correction using equation 1. In the end, all the non-common (spurious)

296 triggers were removed (green trigger in the $R T$ flow and red triggers in the $S T$ flow), and the output

297 subsequence contained only the common triggers which were matched by the LCS algorithm, in which the

298 first and last triggers of these subsequences were the "start trigger" (120) and the "end trigger" (96)

299 respectively. 
Synchronization of acquisition devices in neuroimaging:

An application using co-registration of eye movements and electroencephalography

\begin{tabular}{|c|c|c|c|c|c|c|c|c|c|c|c|c|c|c|c|c|c|c|c|c|c|c|c|c|c|c|c|c|c|c|c|c|}
\hline \# & 1 & 2 & 3 & 4 & 5 & 6 & 7 & 8 & 9 & 10 & 11 & 12 & 13 & 14 & 15 & 16 & 17 & 18 & 19 & 20 & 21 & 22 & 23 & 24 & 25 & 26 & 27 & 28 & 29 & 30 & 31 & 32 \\
\hline RT & 100 & 120 & 201 & 110 & 10 & 11 & & 161 & & 90 & 94 & 110 & 10 & 11 & & 165 & 90 & 95 & 110 & 10 & 11 & & 166 & & 90 & 95 & 110 & 10 & 11 & 164 & 90 & 96 \\
\hline ST & & 120 & 201 & 110 & 10 & 11 & 171 & 161 & 251 & 90 & 94 & 110 & 10 & 11 & 175 & 165 & 90 & 95 & 110 & 10 & 11 & 175 & 166 & 254 & 90 & 95 & 110 & 10 & 11 & 164 & 90 & 96 \\
\hline & & & 2 & & 10 & 11 & & & & 0 & 9 & 110 & 10 & 1 & & 165 & 90 & 95 & 110 & 10 & 11 & & 166 & & 90 & 95. & 110 & 10. & 11 & 164 & 90 & 96 \\
\hline
\end{tabular}

Figure 3. Illustration of the "Longest Common Subsequence" algorithm. $R T$ is the reference sequence of triggers from the reference device. $S T$ is a secondary sequence of triggers from a secondary device. $A L L$ is the longest common subsequence of triggers obtained at the output of the algorithm. The trigger in green is the trigger "missed" by the secondary acquisition device, and triggers in red correspond to the spurious triggers introduced by the secondary acquisition device.

\section{Processing Pause/Resume mode}

As already listed above, in some experimental situations pauses are needed during recording.

310 Acquisition phases are therefore separated by pauses which ensure that synchronization errors do not have

311 a theoretical bound situated between $\left[-R_{T}, R_{T}\right]$ (in practice, it is slightly more, so that round-off errors can

312 be taken into account).

Most acquisition devices have a Pause/Resume function to allow acquisition to be turned off when

314 the recording of the analog signals is not useful, significantly reducing the size of stored files, and

315 consequently shortening data processing time. These pauses can either be scheduled between each part of

316 the experiment to allow participants to rest, or when a technical problem occurs. However, this very useful

317 function can also generate unwanted effects: for instance, if acquisition includes one or more pauses, clock

318 drift will no longer be linear. Consequently, a unique linear regression to fit the alignment of trigger

319 timestamps from beginning to end of acquisition is not efficient. Instead, a piecewise linear regression is

320 required, and a shift correction is introduced, as will be explained below.

Figure 4 below represents a simulated acquisition, containing three acquisition phases (segments

$322 G A, B^{\prime} C^{\prime}$ and $D^{\prime} E^{\prime}$ ), separated by two pauses (segments $A B^{\prime}$ and $C^{\prime} D^{\prime}$ ). In this figure, the drift between the

323 two clocks has been deliberately oversized for clarity. The ideal regression line for the time conversion

324 between the two devices is the segment $G G^{\prime}$ (solid black plain line) with a slope equal to the nominal 
Synchronization of acquisition devices in neuroimaging:

An application using co-registration of eye movements and electroencephalography

325 unitary ratio $\left(R_{T}=1\right)$ of the sampling rates. Assuming that the secondary device (ACQD2) is slightly

326 slower than the reference device (ACQD1), the segment GE (dotted black line) is situated below the ideal

$327 G G^{\prime}$ segment with a unitary slope $\left(R_{T}=1\right)$, and the gap between the slopes of these two segments

328 expresses the amount of clock drift between the two acquisition devices.

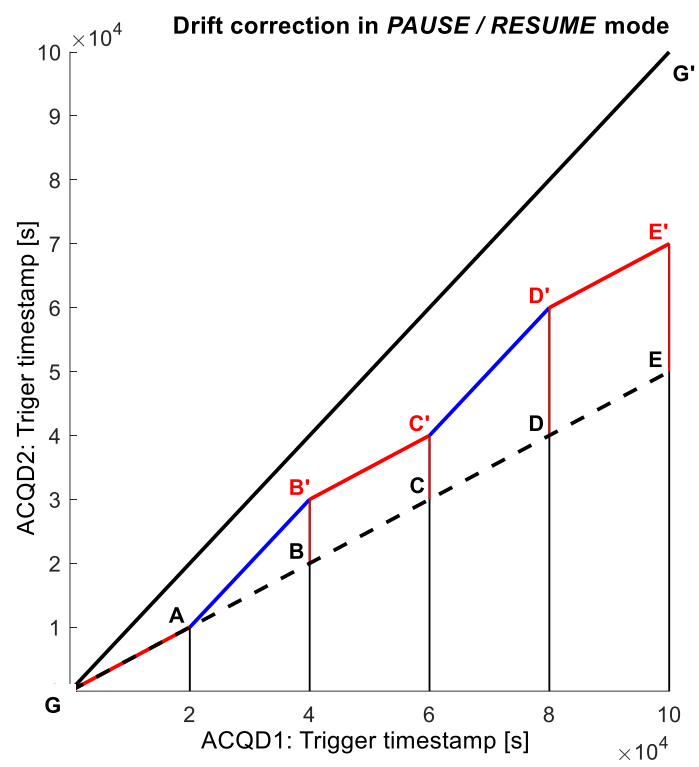

330 Figure 4. Clock drift correction when the Pause / Resume mode is used. Illustration of the theoretical alignment of the trigger timestamps $[\mathrm{s}]$ in a simulation with three acquisition phases $\left(G A, B^{\prime} C^{\prime}, D^{\prime} E^{\prime}\right.$ in red) and two pause phases $\left(A B^{\prime}, C^{\prime} D^{\prime}\right.$ in blue). The segment $G G^{\prime}$ corresponds to the ideal regression line, with $F_{1}=F_{2}$, and the segment $G E$ below illustrates the clock drift between the two devices when pauses are not taken into consideration.

Our reconstruction procedure is based on two assumptions. Firstly, during the acquisition phases,

337 the timestamps of samples and triggers are linearly distributed following the slope of the segment $G E$.

338 Consequently, segments $G A, B^{\prime} C^{\prime}$ and $D^{\prime} E^{\prime}$ representing the acquisition phases are parallel to the segment

$339 G E$. Secondly, during the pause phases, only the duration of the pause and the nominal sampling rate $\left(R_{T}\right)$

340 of the acquisition devices are known. Because synchronization is carried out off-line, the duration of each

341 pause is easily estimated from the timestamp of the last sample before each pause and the timestamp of the

342 first sample after each pause. Consequently, segments $A B^{\prime}$ and $C^{\prime} D^{\prime}$ representing the pause phases are 
Synchronization of acquisition devices in neuroimaging:

An application using co-registration of eye movements and electroencephalography

343 parallel to segment $G G^{\prime}$. Thus, the principle of the supplementary mechanism of shift correction is as

344 follows:

345 - The segments representing the acquisition phases after the first pause (i.e., $B^{\prime} C^{\prime}$ and $D^{\prime} E^{\prime}$ ) should be 346 translated vertically to match the global acquisition segment $G E$.

347 - If the secondary acquisition device is slower (as it is the case in the illustration), a number of 348 samples corresponding to segments $B B^{\prime}$ and $D D^{\prime}$ should be subtracted to correct this effect 349 (conversely a number of samples should be added if the secondary device is faster).

Consequently, the amount of shift correction (length of $B B^{\prime}$ and $D D^{\prime}$ ) does not depend on the pause 352 position in the acquisition session but is only proportional to pause durations.

A one-hour acquisition was simulated, with three acquisition phases, separated by two pauses with 355 position and duration randomly chosen (147 s and $230 \mathrm{~s}$ respectively). In this simulation, there were 3252 356 common triggers with 1071, 1085 and 1096 triggers respectively for the first, second and third acquisition 357 phases. The acquisition devices had the same nominal sampling rate $\left(1 \mathrm{kHz}, R_{T}=1\right)$ and were affected 358 individually by a random clock drift of under $1 \%$ o $(F 1=1000.114 \mathrm{~Hz}$ for the first device and $F 2=$ $359999.869 \mathrm{~Hz}$ for the second one). By applying a unique alignment of the timestamps, the histogram of 360 alignment errors $\left(\varepsilon_{S}\right)$ was multimodal (Figure $5 A$ ) with three modes corresponding to the three acquisition 361 phases. To compensate for the gaps after each pause, the 2 nd and 3rd acquisition segments must be shifted 362 by a certain number of samples, which have to be estimated by the shift correction procedure. In this 363 example, the clock drift between the two devices was $F 1-F 2=1000.114-999.869=0.245 \mathrm{~Hz}$.

364 This would theoretically provide a shift of $0.245 \times 147=36.015$, rounded down to 36 samples after the 365 first pause (duration $147 \mathrm{~s}$ ), and a shift of $0.245 \times(147+230)=92.365$ rounded down to 92 samples 
Synchronization of acquisition devices in neuroimaging:

An application using co-registration of eye movements and electroencephalography

366 after the second pause (duration 230 s). These results are supported by the fact that these shift values (36

367 samples and 92 samples) corresponded exactly to the shift between the modes' positions in the histogram in

368 Figure 5A: $\Delta C_{1}=36$ samples and $\Delta C_{1}+\Delta C_{2}=92$ samples.
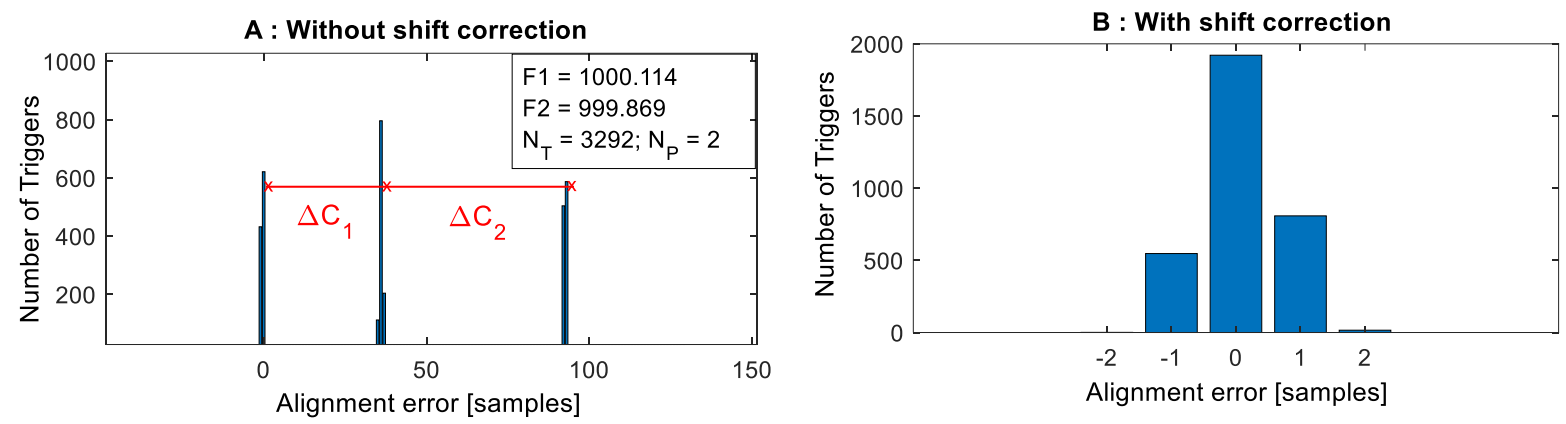

Figure 5. A. Histogram of alignment errors $\left(\varepsilon_{s}\right)$ before shift correction. B. Histogram of alignment errors $\left(\varepsilon_{S}\right)$ after shift correction. Key: $F 1$ - ACQD1 sampling rate-; $F 2$ - ACQD2 sampling rate-; $N_{T}$ - number of triggers-; $N_{P}$ - number of pauses-.

We will now describe in greater detail the implemented algorithm for the drift and shift corrections acquisition phase) toward the real regression line. Two objective criteria stop this iterative process. The first one is the value of the coefficient of determination $R^{2}$ of the regression. It reaches its maximum when the acquisition segments are aligned. The second criterion is derived from the shape of the distribution of alignment errors. This means that the corresponding histogram should be as narrow as possible (not more than $2 . R_{T}+1$ bins), as symmetrical as possible, and centered on bin 0 . The value of the correction is found at the end of the iterations. This correction value shifts all the trigger indexes belonging to this second segment of acquisition. This process is repeated for the third acquisition phase to estimate the second 
Synchronization of acquisition devices in neuroimaging:

An application using co-registration of eye movements and electroencephalography

387 regression is performed, and the slope of the final regression line corresponds to the average slope of 388 segments $G A, B^{\prime} C^{\prime}$ and $D^{\prime} E^{\prime}$. Assessment of the procedure is carried out from the final coefficient of 389 determination $R^{2}$ and from the observed histogram of alignment errors.

390 The coefficient of determination $R^{2}$ was very close to 1 and the estimated value of the shift 391 correction after the first pause was 43 samples (exactly the expected value). After the second pause, the 392 estimated value of the shift correction was 110 samples (the expected value was 109 samples). The 393 positions of the triggers for the secondary device were then linearly aligned on the time positions given by 394 the sampling of the reference device. This was confirmed by the histogram of alignment errors (Figure 5B), 395 which was, at this point, mainly described by three bins (positions $-1,0,1$ ). The theoretical bounds were $396\left[-R_{T}, R_{T}\right]$ given $[-1,1]$ for $R_{T}=1$. We also observed non-null values in the extreme bins of the histogram 397 (positions -2 and +2 ). These values were due to the unavoidable jitters of the sampling rates and to the 398 round-off errors.

In short, the proposed synchronization is composed of three steps: (i) the LCS algorithm, as 401 described in the "Processing missing and spurious triggers" section, used to obtain the sequence of all 402 common triggers detected by the acquisition devices, (ii) the clock drift correction with a piecewise linear 403 regression for temporal alignment during each acquisition segment and the shift estimation for each pause 404 phase, and finally (iii) a linear regression for complete temporal alignment after the application of shift 405 corrections. Validation on real data

In order to verify the effectiveness of our synchronization procedure, we tested it on real data (see 408 "Datasets" section below). The assessment of its quality is illustrated in the "Synchronization procedure 
Synchronization of acquisition devices in neuroimaging:

An application using co-registration of eye movements and electroencephalography

409 quality assessment" section, and the comparison with another available implementation (EYE-EEG

410 toolbox) in the "Comparison with the EYE-EEG toolbox" section.

411

412 Datasets

413 Data came from three different experiments (Frey, Ionescu, Lemaire, Lopez-Orozco, Baccino \& Guérin-

414 Dugué, 2013; Devillez, Guyader \& Guérin-Dugué, 2015; Van Humbeeck, Meghanathan, Wagemans,

415 Leeuwen \& Nikolaev, 2018). The first two datasets came from our own records, and the third was provided

416 by another laboratory, in order to validate our method using an independent dataset.

417

All three experimental designs used an eye-tracker (ACQD1) and EEG system (ACQD2) with the

418 same setups shown in Figure 1. The eye-tracker was the same in all experiments (Eyelink 1000; SR

419 Research), with a $1000 \mathrm{~Hz}$ sampling rate for the first two, and $250 \mathrm{~Hz}$ for the third. EEG signals were

420 sampled at $1000 \mathrm{~Hz}$ in the first experiment (32-channel BrainAmp ${ }^{\mathrm{TM}}$ system, Brain Products GmbH), at

$4211200 \mathrm{~Hz}$ in the second experiment (32-channel GAMMAsys gtec system, G.tec, Inc.), and at $250 \mathrm{~Hz}$ in the

422 third (256-channel Electrical Geodesics System, Electrical Geodesics Inc., Eugene, OR). The eye-tracker

423 was the reference device for the synchronization procedure, and the EEG system was the secondary device.

$424 \quad$ In Frey et al. (2013), nineteen participants took part in the experiment, with 180 trials per

425 participant. For nine participants, the whole acquisition phase was carried out without using the

426 Pause/Resume mode (one acquisition segment). For eight participants, the Pause/Resume mode was used

427 once (providing two acquisition segments), and for two participants, there were two pauses (three

428 acquisition segments). These three situations are synthesized in Table 1 and labeled scenarios 1.1, 1.2 and

4291.3 respectively. The Devillez et al. (2015) experiment consisted of a visual search experiment, with thirty-

430 nine participants and two conditions, and sixty trials per condition. Among the seventy-eight available

431 datasets, forty-eight (labeled scenario 2 in Table 1) were randomly selected in order to have the same order 
Synchronization of acquisition devices in neuroimaging:

An application using co-registration of eye movements and electroencephalography

432 of magnitude of the total number of triggers recorded as with scenario 1 datasets. Finally, one dataset was

433 extracted from the third experiment (Van Humbeeck et al., 2018), in which twenty-three participants

434 carried out a contour integration task. This dataset appears in Table 1, and is entitled scenario 3. In

435 scenarios 2 and 3, the Pause/Resume mode was not used and there was only one acquisition segment per

436 recording.

437

\begin{tabular}{llllll}
\hline Scenario & 1.1 & 1.2 & 1.3 & 2 & 3 \\
\hline EEG system & BrainAmp & BrainAmp & BrainAmp & GTec & EGI \\
EEG sampling rate [Hz] & 1000 & 1000 & 1000 & 1200 & 250 \\
ET sampling rate [Hz] & 1000 & 1000 & 1000 & 1000 & 250 \\
\# Datasets & 9 & 8 & 2 & 48 & 1 \\
\# Segments (\# Pauses) & $1(0)$ & $2(1)$ & $3(2)$ & $1(0)$ & $1(0)$ \\
\# Common triggers & 11235 & 9916 & 2413 & 26578 & 482 \\
\# Missing triggers & $0(0 \%)$ & $0(0 \%)$ & $0(0 \%)$ & $0(0 \%)$ & $968(66.8 \%)$ \\
\# Spurious triggers & $2678(19.2 \%)$ & $2359(19.2 \%)$ & $622(20.5 \%)$ & $0(0 \%)$ & $0(0 \%)$
\end{tabular}

438

439

440

441

442

443

444

445

446

447

Table 1. Dataset description. Keys: The five different datasets are labeled scenarios 1.1, 1.2, 1.3, 2 and 3. Each is described with the type of EEG system, the EEG sampling rate, the eye-tracker (ET) sampling rate, the number of datasets, the number of acquisition segments, the number of pauses, the cumulative number of the common triggers for all datasets, of the missing triggers and of the spurious triggers detected in the EEG data flow. The percentages in parentheses were computed in relation to the total number of triggers.

These datasets enabled us to assess the quality of the proposed synchronization procedure in various situations for $R_{T}=1$ (scenarios 1.1, 1.2, 1.3 and 3) and $R_{T}=1000 / 1200$ (scenario 2), including the problem of spurious triggers (scenarios 1.1, 1.2, and 1.3), the problem of missing triggers (scenario 3) and the use of the Pause/Resume mode (scenarios 1.2 and 1.3). A dataset illustrating each of these scenarios is 
Synchronization of acquisition devices in neuroimaging:

An application using co-registration of eye movements and electroencephalography

448 available (Zenodo repository: https://doi.org/10.5281/zenodo.5554071). The datasets were synchronized

449 using our software, named "GazeEEGSynchro", which implements our synchronization proposal and

450 which is also available in the Zenodo repository. A tutorial showing how to use the "GazeEEGSynchro"

451 software is provided in the "Supplementary Material". After synchronization, data from the EEG and eye-

452 tracker recordings were grouped together, and as such became the synchronized data. The synchronized

453 data came from the EEG channels, the vertical and horizontal eye positions, and a "blink" logic signal

454 identifying the onset and the offset of each blink. When the data from the eye tracker were added to the

455 EEG channels from EEG recording, there were, therefore, either three extra channels when recording of

456 ocular data was in monocular mode, and five extra channels when recording of ocular data was in binocular

457 mode.

458

459 Synchronization procedure quality assessment

460 In the following sections, we evaluate the quality of our synchronization procedure in two ways.

461 The first section, "Trigger alignment", is based on the distribution of the alignment errors. The second

462 section, "Blink alignment", is based on blinks, which are easily detected on the eye-tracker, and on EEG

463 signals. This was done to estimate the time delay between blink onsets from the two modalities, throughout

464 the experiment, with and without clock drift correction. Two implementations were performed, one on all

465 scenario 1 datasets, and the other on all scenario 2 datasets. In the latter case, an additional step of

466 resampling the synchronized data was required, as the two devices did not initially have the same sampling

467 rate.

$468 \quad$ Trigger alignment

469 In this section we present the distribution of the alignment errors on the trigger timestamps after clock

470 drift correction from acquisitions using the Pause/Resume mode (scenarios 1.2 and 1.3). To do so, we selected 
Synchronization of acquisition devices in neuroimaging:

An application using co-registration of eye movements and electroencephalography

471 two random datasets, the first from scenario 1.2 with one pause (two acquisition segments), and the second

472 from scenario 1.3 with two pauses (three acquisition segments). The synchronization was applied separately

473 to the two datasets. We expected to observe multimodal distributions of the alignment errors when clock drift

474 correction was applied overall, on the complete acquisition, and monomodal distributions when the shift

475 correction was incorporated into the clock drift correction.

476 Figure $6 \mathrm{~A}$ presents the observed histograms of alignment errors for the first dataset, without shift

477 correction, i.e. when the clock drift correction was applied once on the complete acquisition. The number

478 of alignment errors in this histogram represents the number of common triggers after application of the

479 LCS algorithm. There were 1304 common triggers distributed over two acquisition phases, respectively

480271 for the first phase, and 1033 for the second. As explained previously ("Processing Pause/Resume

481 mode" section), this histogram is composed of two modes corresponding to the two acquisition phases

482 separated by one pause phase. Figure $6 B$ presents the observed histogram of alignment errors, with a shift

483 correction included into the clock drift correction.
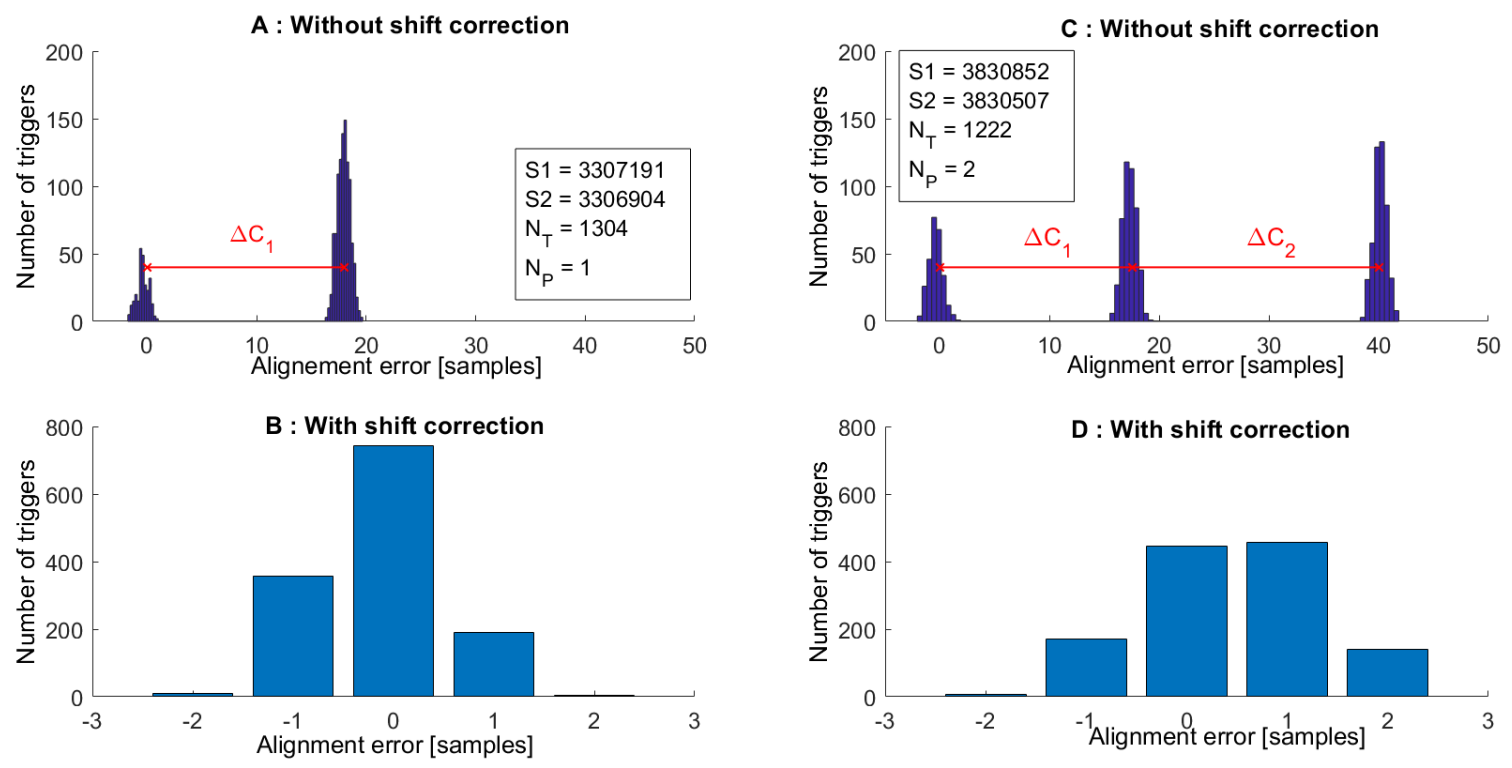
Synchronization of acquisition devices in neuroimaging:

An application using co-registration of eye movements and electroencephalography

486

487

488

489

490

491

492

493

494

495

496

497

498

499

500

501

502

503

504

505

506

507

508

509

510

511

Figure 6. Alignment errors on real data from acquisitions using the Pause/Resume mode, (left plots scenario 1.2; right plots scenario 1.3). A. Histogram of alignment errors $\left(\varepsilon_{\mathrm{s}}\right)$ without shift correction (one pause, two acquisition phases). B. Histogram of alignment errors $\left(\varepsilon_{\mathrm{s}}\right)$ with shift correction (one pause). C. Histogram of alignment errors $\left(\varepsilon_{s}\right)$ without shift correction (two pauses, three acquisition phases). D. Histogram of alignment errors $\left(\varepsilon_{\mathrm{S}}\right)$ with shift correction (two pauses). Key: S1 - number of eye-tracker samples-; $S 2$ - number of EEG samples-; $\mathrm{N}_{\mathrm{T}}$ - number of common triggers-; $\mathrm{N}_{\mathrm{P}}$ - number of pauses-.

Similarly, for the second dataset, the graph presented in Figure 6C illustrates the observed

histogram of alignment errors after clock drift correction but without shift correction. For this dataset, there were 1222 common triggers distributed as follows: 273 triggers during the first acquisition phase, 469 during the second one and 480 during the third one. This histogram is composed of three modes, one for each acquisition phase. See Figure Al in the Appendix for the joint representation of the trigger timestamps in both modalities showing the three acquisition phases and the time shifts between them. Figure $6 D$ presents the observed histogram of alignment errors with a shift correction included into the clock drift correction. The shift value for each pause was estimated by the shift correction procedure as explained in the "Processing Pause/Resume mode" section. For the first dataset with one pause, the estimated applied shift was equal to 17 samples. As Figure $6 A$ shows, this value corresponds to the shift $\Delta C_{1}$ between the two modes. For the second dataset, with two pauses, the estimated applied shift for the second acquisition segment was equal to 17 samples, and for the third acquisition segment, a last estimated shift was equal to 22. As Figure $6 C$ shows, these two values were in line with the shifts observed between the histogram modes $\left(\Delta C_{1}, \Delta C_{2}\right)$. Finally, after clock drift correction with shift correction, the $R^{2}$ coefficient was very close to one 1 for the final linear regression. This meant that the trigger positions of the reference and secondary devices were very well aligned in each synchronized dataset. This was confirmed by the histogram of alignment errors after clock drift correction and with shift correction (Figure 6B, Figure 6D), where the non-null values of the extreme bins of the histogram (position \pm 2 ) were due to the unavoidable jitters of the sampling periods and to errors due to rounding. 
Synchronization of acquisition devices in neuroimaging:

An application using co-registration of eye movements and electroencephalography

\section{Blink alignment}

In order to assess the overall quality of our procedure throughout the entire experiment, we analyzed

515 the temporal delay between common events which were almost simultaneous and easily detectable in both

516 modalities. Using the notation presented in the "Principle of trigger-based synchronization" $\operatorname{section,~} t\left(s_{1}^{(i)}\right)$

517 and $t\left(s_{2}^{(i)}\right)$ represent the onset of the $i^{\text {th }}$ event extracted from the dataset of the first acquisition device (the

518 reference device), and from the dataset of the second acquisition device, respectively. The difference

$519 t\left(s_{1}^{(i)}\right)-t\left(s_{2}^{(i)}\right)$ is the temporal delay for the onset of the same event $i^{\text {th }}$ in both acquisitions. After

520 synchronization, $t\left(s_{21}^{(i)}\right)$ represents the onset of the $\mathrm{i}^{\text {th }}$ event extracted from data recorded by the second

521 acquisition device in the synchronized dataset using the first device as reference. After synchronization

$522 t\left(s_{1}^{(i)}\right)$ becomes the reference time for the onset of the $\mathrm{i}^{\text {th }}$ event. The difference $t\left(s_{1}^{(i)}\right)-t\left(s_{21}^{(i)}\right)$ represents

523 the temporal delay for the onset of the same $\mathrm{i}^{\text {th }}$ event in the synchronized dataset. The former difference is

524 denoted by $\Delta t_{N O}\left(s^{(i)}\right)$, which corresponds to the delay without clock drift correction represented by

$525 \Delta t_{Y E S}\left(s^{(i)}\right)$, which corresponds to the delay with clock drift correction. Without clock drift correction, the

526 delay $\left|\Delta t_{N O}\left(s^{(i)}\right)\right|$ in absolute value should increase along acquisition as the accumulated amount of clock

527 drift. However, since the event is assumed to be almost simultaneous in both modalities, when clock drift

528 correction is applied, the delay $\Delta t_{Y E S}\left(s^{(i)}\right)$ should be relatively small, and in the same order of magnitude

529 as alignment errors.

530 Eye blinks were the ideal candidate for this "almost simultaneous and easily detectable common

531 events" in both modalities. Blinks are generally involuntary acts of shutting and opening the eyelids, and

532 this interrupts the saccade-fixation series. Both blinks and saccades can mask brain activity, because they

533 elicit larger electric potentials than baseline signals (Picton et al., 2000). For our purposes, we used the 
Synchronization of acquisition devices in neuroimaging:

An application using co-registration of eye movements and electroencephalography

534 blinks detected by the eye-tracker as a reference, and those estimated from the EEG signals, throughout the

535 experiment. The time delays between the blink onset in each modality, with and without clock drift

536 correction $\left(\Delta t_{Y E S}(),. \Delta t_{N O}().\right)$ were computed. For all datasets from scenarios 1 and 2 , and without clock

537 drift correction, the two trigger flows were only aligned on the same first common trigger to set the same

538 timestamp (0) for the beginning of recording in both modalities. After that, the synchronization with the

539 complete clock drift correction was applied from the first common trigger up to the last common trigger.

540 This synchronization included a resampling step at $1000 \mathrm{~Hz}$ for EEG signals from scenario 2 (initial EEG

541 sampling rate at $1200 \mathrm{~Hz}$, see Table 1) in order to obtain the same sampling rate as the reference device

542 (eye-tracker at $1000 \mathrm{~Hz}$ ). Resampling was not performed on datasets from scenariol because the two

543 devices had the same sampling rate of $1000 \mathrm{~Hz}$ (see Table 1). The scenario 3 was not used for this

544 assessment because there was only one dataset and the configuration with the same sampling rate was

545 already considered with scenario 1 datasets.

546 The logic Blink signal (True during blink, False otherwise) was used to time-lock the EEG signal on

547 the FP1 channel which was epoched within a temporal window of $[-0.5 ; 0.5]$ seconds on each side of the

548 blink onset. The number of epochs was therefore equal to the number of blinks. Blink duration was

549 estimated from the duration of the logic Blink signal, and only epochs with blinks lasting between 100 and

$550400 \mathrm{~ms}$ were retained for analysis: 2507 epochs from scenario 1 and 4919 from scenario 2, which on

551 average corresponded, respectively to 132 and 102 epochs per participant. On the EEG signal, blinks were

552 detected on the FP1 channel (Jung, Makeig, Westerfield, Townsend, Courchesne \& Sejnowski, 2000), and

553 were related to the local maximum value of the first derivative, computed with a Canny-Deriche Filter

554 (Deriche, 1990). The parameter $\alpha$ of the filter was calculated so that: $\alpha \cdot \sigma=2.5 / \sqrt{\pi}$ where $\sigma$ is the width

555 of the Gaussian filter. To ensure accurate detection, the following values were empirically set: $\sigma=10$ and $556 \alpha=0.14$ 
Synchronization of acquisition devices in neuroimaging:

An application using co-registration of eye movements and electroencephalography

Figure 7 illustrates five epochs (index 2, 30, 60, 89, 118) distributed across the experiment (towards

558 the beginning for the first epochs, and towards the end for the last epochs), for a given participant. The first

559 two dotted lines represent the FP1 signal and its derivative without clock drift correction. The Blink signal

560 is represented on the third line. The fourth and the fifth plain lines represent the same FP1 signal and its

561 derivative after clock drift correction.

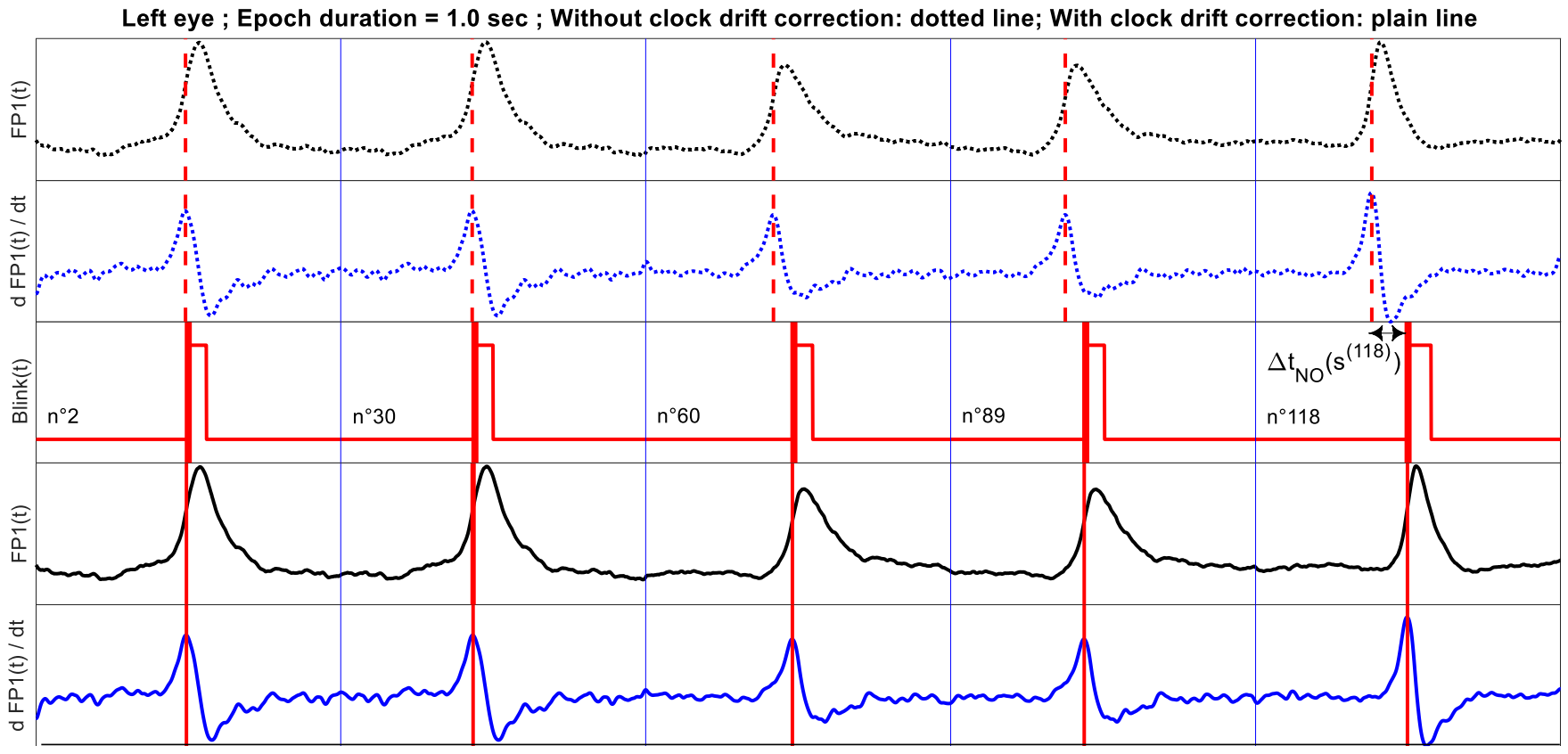

Figure 7. Illustration of the clock drift correction observed on eye blink events. The vertical blue lines delimit the end of each epoch. Selected channel: FP1 from EEG data and left eye blink from eye-tracker data. Upper two signals: channel FP1 and its first derivative without clock drift correction. Middle signal: logic Blink signal. Lower two signals: channel FP1 and its first derivative after clock drift correction. The vertical dashed red markers indicate blink detection on the EEG signal $(F P 1(t))$ from the first derivative $d F P 1(t) / d t$ on the dotted line without clock drift correction. For epoch number 118 (i.e. the $188^{\text {th }}$ blink), $\Delta t_{N O}\left(s^{(118)}\right)$ is the delay for the $188^{\text {th }}$ blink onset between both modalities without clock drift correction. The vertical plain red markers indicate blink detection on the EEG signal $(F P 1(t))$ from the first derivative $d F P 1(t) / d t$ in plain line with clock drift correction.

In Figure 7, in the absence of correction, clock drift is clearly visible from the increasing length of 
Synchronization of acquisition devices in neuroimaging:

An application using co-registration of eye movements and electroencephalography

576 the blinks detected on the EEG signal (the vertical dashed red line indicates the maximum value of the first

577 derivative on the dotted line). These delays are represented by $\Delta t_{N O}\left(s^{(2)}\right), \Delta t_{N O}\left(s^{(30)}\right), \Delta t_{N O}\left(s^{(60)}\right)$,

$578 \Delta t_{N O}\left(s^{(89)}\right), \Delta t_{N O}\left(s^{(118)}\right)^{5}$ and qualitatively $\Delta t_{N O}\left(s^{(2)}\right)<\Delta t_{N O}\left(s^{(30)}\right)<\Delta t_{N O}\left(s^{(60)}\right)<\Delta t_{N O}\left(s^{(89)}\right)<$

$579 \Delta t_{N O}\left(s^{(118)}\right)$. However, when clock drift correction is applied, these delays (i.e., delay between the vertical 580 plain red lines on the Blink signal, and the vertical plain red line indicating the maximum value of the first 581 derivative in plain line) are so short that they are not visible in Figure 7.

582 In order to quantify these delays in scenarios 1 and 2, without and with clock drift correction, we 583 computed the delays $\Delta t_{N 0}\left(s^{(i)}\right)$ and $\Delta t_{Y E S}\left(s^{(i)}\right)$ for all epochs. In this way, each blink was characterized by 584 three features (all in ms): (1) its absolute timestamp in the experiment, i.e. its onset given by the eye-tracker $585 t\left(s_{1}^{(i)}\right)$ as the reference, $(2)$ the corresponding delay $\Delta_{N O}\left(S^{(i)}\right)$ without clock drift correction, and (3) the 586 corresponding delay $\Delta_{Y E S}\left(s^{(i)}\right)$ with clock drift correction. Figure 8 left shows the joint distributions of $587 \Delta_{N O}$ vs $t$ (red dots) and $\Delta_{Y E S}$ vs $t$ (green dots) for data from scenario 1 (Top) and for data from scenario 2 588 (Bottom).

\footnotetext{
${ }^{5}$ Because this delay is quite long, it is visible in Figure 7.
} 
Synchronization of acquisition devices in neuroimaging:

An application using co-registration of eye movements and electroencephalography

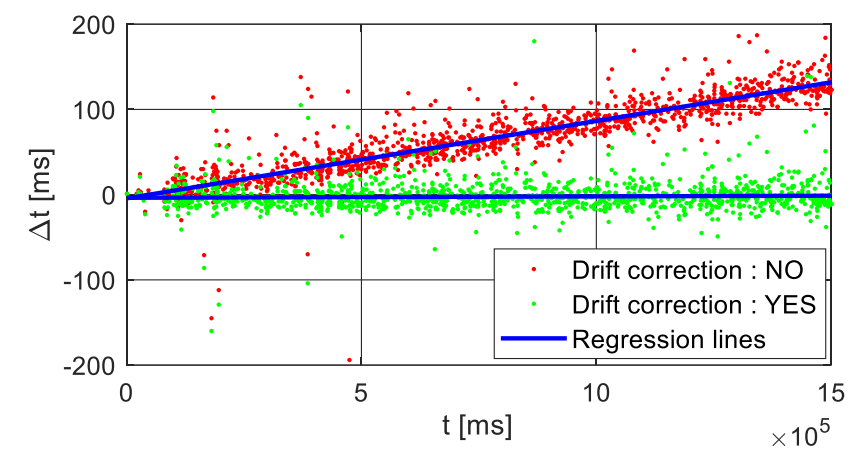

Scenario 1
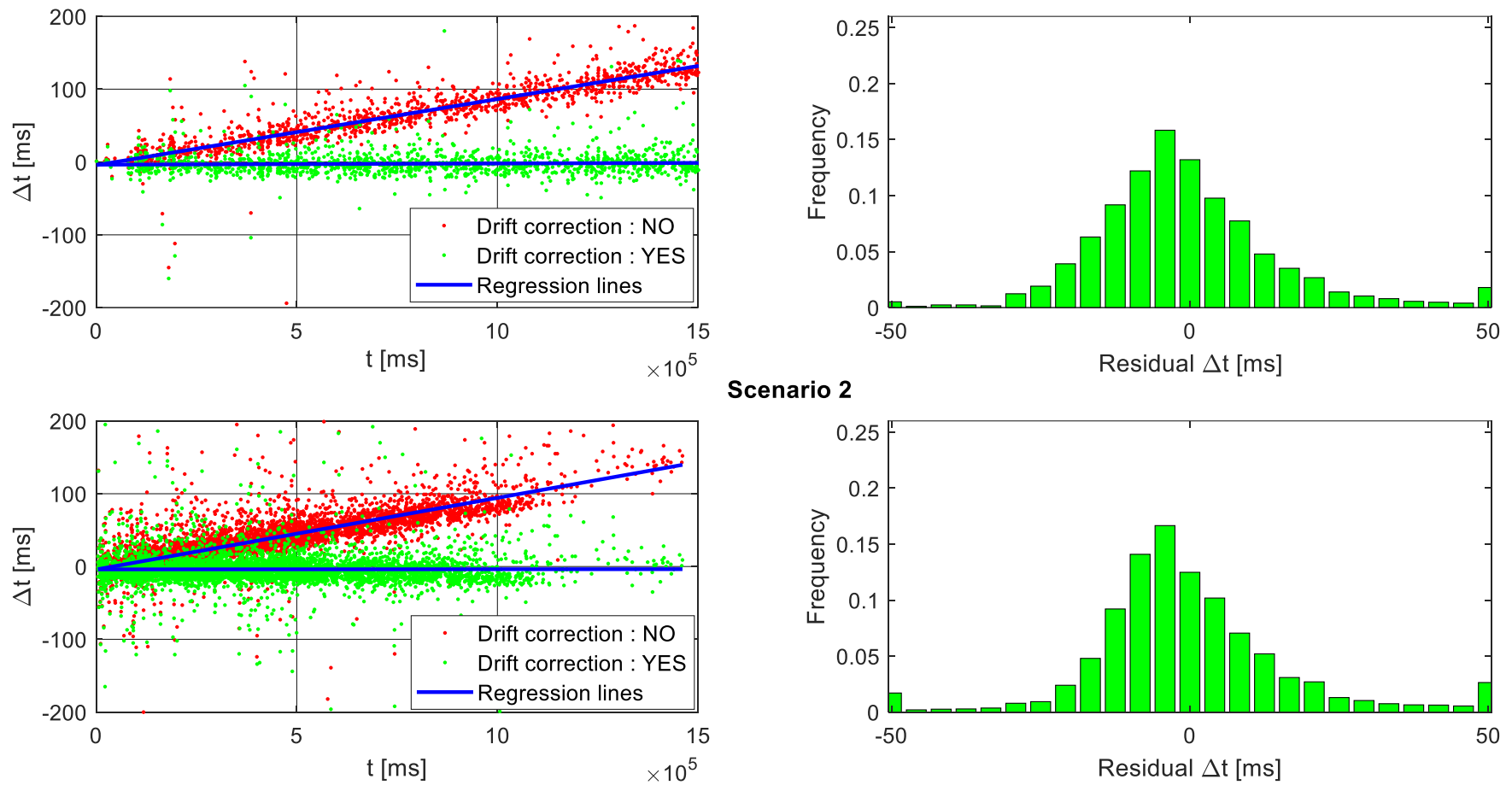

Scenario 2

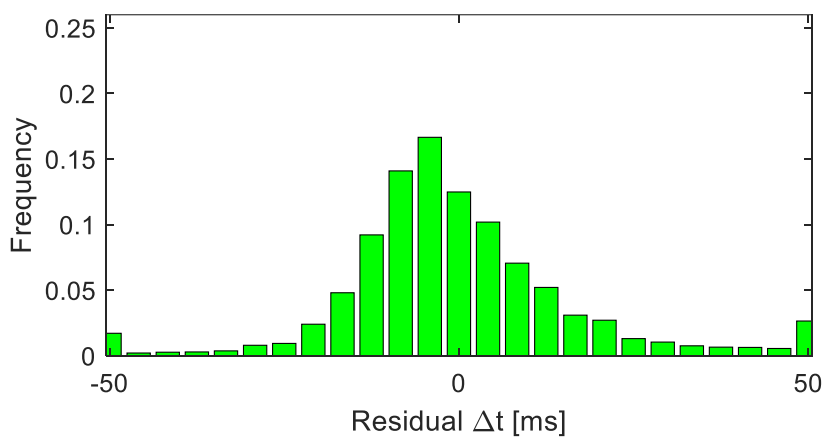

Figure 8. Statistical analysis of blink detection delay. Left: joint representation of the eye blinks collected from scenario 1 (Top) and from scenario 2 (Bottom). The red dots represent the $\Delta_{N O}$ data (without drift correction) while the green dots represent the $\Delta_{Y E S}$ data (with the drift correction procedure). Time on the horizontal axis is expressed in milliseconds. Right: Histogram of the residual errors between $\Delta_{Y E S}$ and the values predicted by the linear regression, from scenario 1 (Top) and from scenario 2 (Bottom). The extreme bins of the histograms correspond to "less than $-50 \mathrm{~ms}$ " and "more than $50 \mathrm{~ms}$ ".

As expected, and plotted separately for the two scenarios on Figure 8 left, a linear regression model 598 attempts to fit the linear relationship between the $\Delta_{N 0}$ or $\Delta_{Y E S}$ delays as a function of the absolute 599 timestamp $t$ of the blink onset. The two regression lines are represented in blue on Figure 8 left, and their 600 slope $(a)$ and intercept $(b)$ are given in Table 2. Figure 8 right shows the histograms of residual errors, i.e., 601 the differences between the observed values of delay $\Delta_{Y E S}$ and the values predicted by the linear regression 602 line. The mean and standard deviation (in brackets) of these distributions were -1.34 (39.83) ms for the first 603 scenario, and $-3.83(35.07) \mathrm{ms}$ for the second one. 
Synchronization of acquisition devices in neuroimaging:

An application using co-registration of eye movements and electroencephalography

\begin{tabular}{lccc}
\hline & Epoch number & $\Delta_{N O}$ & $\Delta_{Y E S}$ \\
& 23564 & $\mathrm{a}=8.8901 .10^{-5}$ & $\mathrm{~m}$ \\
& & $\mathrm{~b}=0.1291 \mathrm{~ms}$ & $\mathrm{a}=3.3520 .10^{-7}$ \\
& & $\mathrm{~b}=0.2268 \mathrm{~ms}$ \\
Scenario 1 & & \\
& & $\mathrm{a}=9.5372 .10^{-5}$ & $\mathrm{a}=-2.265210^{-6}$ \\
& $\mathrm{~b}=0.8523 \mathrm{~ms}$ & $\mathrm{~b}=0.7970 \mathrm{~ms}$
\end{tabular}

605

606 607

608

609

610

611

612

613

614

615

616

617

618

619

620

Table 2. Slope $(a)$ and intercept $(b)$ of the linear regression between the dependent variable $\left(\Delta_{N O}\right.$ or $\left.\Delta_{Y E S}\right)$ and the independent variable $t$ representing the blink onset given by the eye-tracker.

We observed similar values for the slopes $(a)$ in scenarios 1 and 2 , whereas the synchronization for the latter included a supplementary resampling step at $1000 \mathrm{~Hz}$ for EEG signals. More importantly, we observed a decrease of two orders of magnitude between the slopes $(a)$ without and with drift correction. Interpretation of slope $a$ seems straightforward. In both scenarios, for example, the two slopes were 8.8901. $10^{-5}$ and $9.5372 .10^{-5}$ ie. about $10.10^{-5}$, when clock drift was not corrected. With such a value, and for an acquisition time of $15.10^{5} \mathrm{~ms}$, the synchronization delay is about $150 \mathrm{~ms}$ (Figure 7). Thus, for a one-hour experiment $\left(36.10^{5} \mathrm{~ms}\right)$, the progressive desynchronization time between the two data flows would come to $360 \mathrm{~ms}$ at the end of the experiment. This shift value is clearly not negligible, and in the context of our EFRP data, could lead to overlapping and/or misinterpretation of the resulting EEG components. After synchronization, a slope of around $10^{-6}$ can be expected. Consequently in a one-hour experiment, progressive residual desynchronization would be reduced by 100 (around $3.6 \mathrm{~ms}$ ), which corresponds to a negligible number of samples. 
Synchronization of acquisition devices in neuroimaging:

An application using co-registration of eye movements and electroencephalography

621 Comparison with the EYE-EEG toolbox

We applied our method implemented in GazeEEGSynchro software, and the synchronization

623 method implemented in the EYE-EEG environment (Dimigen et al., 2011), to datasets from scenarios 2 and

6243 in order to compare their respective main functionalities, overall quality and time processing capacities.

625 Indeed, the scenario 2 had the largest number of datasets.

626

627

628

629

630

631

632 section).

633

634

635

636

637

638

639

640

641 Common triggers: selection and regression

642

643

\section{Input/Output information} choose the reference device.
Both procedures require the eye-tracker and the EEG files as inputs. However, for the EYE-EEG method, the names of the two common synchronization triggers have to be entered, one indicating the beginning of acquisition, and the other the end. These inputs are not necessary with our solution because of the alignment of triggers provided by the LCS algorithm (cf. "Processing missing and spurious triggers"

Regarding output, the interface can be used to select which ocular channel needs to be added with the EYE-EEG method. With our implementation, all ocular channels are added, and after synchronization, users can remove unnecessary channels as required.

In the EYE-EEG method, the reference device is always the EEG device. This means that synchronized data and triggers are sampled at the same frequency as the EEG device. As a result, only the ocular channels are interpolated on the EEG sampling timestamps. With our implementation, users can

In our method, common triggers are automatically detected by the LCS algorithm. The start and end triggers are directly identified as the first and last common triggers, respectively. Only data from EEG and 
Synchronization of acquisition devices in neuroimaging:

An application using co-registration of eye movements and electroencephalography

644 eye-tracker recordings between these two timestamps are synchronized, either on the EEG timeline or on

645 the eye-tracker timeline, depending the user's choice of reference device.

646 In the EYE-EEG method, users have to provide the start and end triggers. Common triggers are then

647 searched for between these two triggers, and are determined in two steps. The first step is the intersection

648 of the trigger lists in each modality. Spurious triggers and missing triggers are therefore naturally excluded

649 from this common list. The second step consists of finding which of these triggers can be matched in both

650 modalities according to their timestamps. The eye-tracker timestamps of the start and end triggers are

651 arranged linearly to match with the corresponding triggers in the EEG timeline. After this linear

652 transformation from the eye-tracker timestamps to EEG timestamps, the matching of each common trigger

653 is performed if the gap between the original timestamp on the EEG timeline and the transformed timestamp

654 is smaller than a given tolerance (four samples, default value of this parameter). One single linear

655 regression is performed on the timestamps of each common trigger in order to globally optimize the

656 timestamps of the common triggers and to find the linear relationship between the EEG timeline and the

657 eye-tracker timeline.

658 We have shown that a piecewise linear regression needs to be carried out for acquisition in

659 Pause/Resume mode, and this functionality is not implemented in the EYE-EEG toolbox. Consequently,

660 synchronization of a scenario 1.3 dataset containing two pauses (Table 1, Figure 6 C D) was not successful

661 with the Eye-EEG method (Figure A2 in the Appendix).

662 Finally, Table 3 summarizes the main differences between both methods according to their

663 functionalities.

Proposed synchronization EYE-EEG

method

Implementation

DOS application written in

Matlab ${ }^{\circledR}$ functions integrated into

$\mathrm{C}++$

EEGLab 
Synchronization of acquisition devices in neuroimaging:

An application using co-registration of eye movements and electroencephalography

\begin{tabular}{|c|c|c|}
\hline Eye-tracker & SR Research & $\begin{array}{l}\text { SR Research, SMI, Tobii, Tobii } \\
\text { Pro }\end{array}$ \\
\hline$\overline{\mathrm{EEG}}$ & $\begin{array}{l}\text { Converted to BrainVision } \\
\text { format }^{6}\end{array}$ & $\begin{array}{l}\text { EEG format supported by } \\
\text { EEGLAB }\end{array}$ \\
\hline Reference device & Eye-tracker or EEG & EEG \\
\hline Ocular channels & All & Selected by user \\
\hline $\begin{array}{l}\text { Trigger identification: } \\
\text { begin / end recording }\end{array}$ & Automatic & Selected by user \\
\hline $\begin{array}{l}\text { Recording with Pause/Resume } \\
\text { mode }\end{array}$ & Yes & No \\
\hline Evaluation from alignment errors & & \\
\hline $\begin{array}{l}\text { - Alignment errors for all } \\
\text { common triggers }\end{array}$ & In text format (log file) & $\begin{array}{l}\text { Not directly provided, but can be } \\
\text { computed from available variables }\end{array}$ \\
\hline - Global histogram & In text format (log file) & \\
\hline $\begin{array}{l}\text { - Specific histogram for each } \\
\text { trigger type }\end{array}$ & In text format (log file) & $\begin{array}{l}\text { In stored variables and in graphic } \\
\text { Not provided }\end{array}$ \\
\hline $\begin{array}{l}\text { - Overview of event latencies in } \\
\text { output datasets }\end{array}$ & Not provided & Graphic \\
\hline $\begin{array}{l}\text { - Regression between event } \\
\text { latencies }\end{array}$ & Coefficient of determination & $\begin{array}{l}\text { Graphic and coefficient of } \\
\text { determination }\end{array}$ \\
\hline - Trigger and event counting & Provided & Provided \\
\hline Evaluation from cross-correlation & $\begin{array}{l}\text { Must be implemented by user } \\
\text { in their preferred environment }\end{array}$ & In stored variables and in graphic \\
\hline
\end{tabular}

Table 3. Comparison of the main functionalities of both methods. 
Synchronization of acquisition devices in neuroimaging:

An application using co-registration of eye movements and electroencephalography

\section{Overall quality}

The quality assessment was performed on the scenario 3 dataset for illustration, and on scenario 2 669 datasets to provide statistical comparisons.

670 Each type of synchronization software provided a histogram of alignment errors after 671 synchronization, expressed in number of samples. Each histogram (Figure 9) was computed on 11 bins, 672 from -5 samples up to 5 samples. For each histogram, the following statistical features were computed:

673 - Position: mean (best when close to zero),

$674 \quad$ - Spread: standard deviation (best when close to zero),

675 - Flatness: Kurtosis coefficient (best when high),

$676 \quad$ - Asymmetry: Skewness coefficient (best when close to zero).

677

678

The EYE-EEG method provided better alignment errors for the scenario 3 dataset than our proposed

679 method, according to the statistical features computed on the distribution of alignment errors. The

680 corresponding histograms are illustrated in Figure 9: "position" closest to zero (0.035 vs 0.475), better

681 “spread" (0.317 vs 0.449), better "flatness" (9.663 vs 4.436) and better "asymmetry" (0.111 vs 1.058$)$. 
Synchronization of acquisition devices in neuroimaging:

An application using co-registration of eye movements and electroencephalography

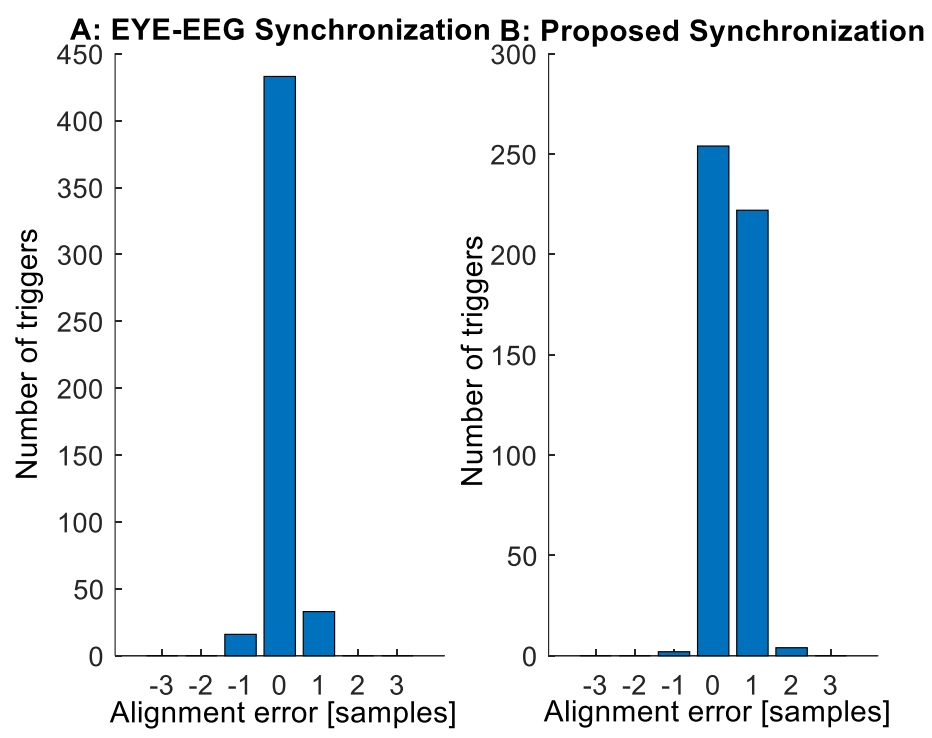

684 Figure 9. Histogram of alignment errors after synchronization for the scenario 3 dataset, for (A) the EYE685 EEG synchronization and (B) the proposed synchronization. The counts for bins $-5,-4,4$ and 5 were zero 686 and were not represented.

Dimigen et al. (2011) first introduced cross-correlation to assess the quality of ocular artefact 689 reduction in EEG signals, and of synchronization. For this later, the cross-correlation was computed 690 between the horizontal position of the eye, and the difference between the amplitudes recorded on the right 691 and left electro-oculogram (horizontal EOG) channels. Segments featuring blinks and signals that were too 692 noisy were removed beforehand. The cross-correlation was computed with the "xcorr" function provided 693 by the "Signal Processing" toolbox in Matlab®. Figure 10 A B illustrates the two signals obtained from a randomly-selected trial, and Figure $10 \mathrm{C}$ 695 D shows the cross-correlation result. The position of the maximum value of the cross correlation must be 696 close to zero, and lag is defined by the time position of this maximum value. A positive lag means that the 697 transition of the horizontal eye position occurs later than the transition in the EOG signals. For this dataset, 698 the cross-correlation reached its maximal value for the same lag length (3 samples) in both methods. 
Synchronization of acquisition devices in neuroimaging:

An application using co-registration of eye movements and electroencephalography
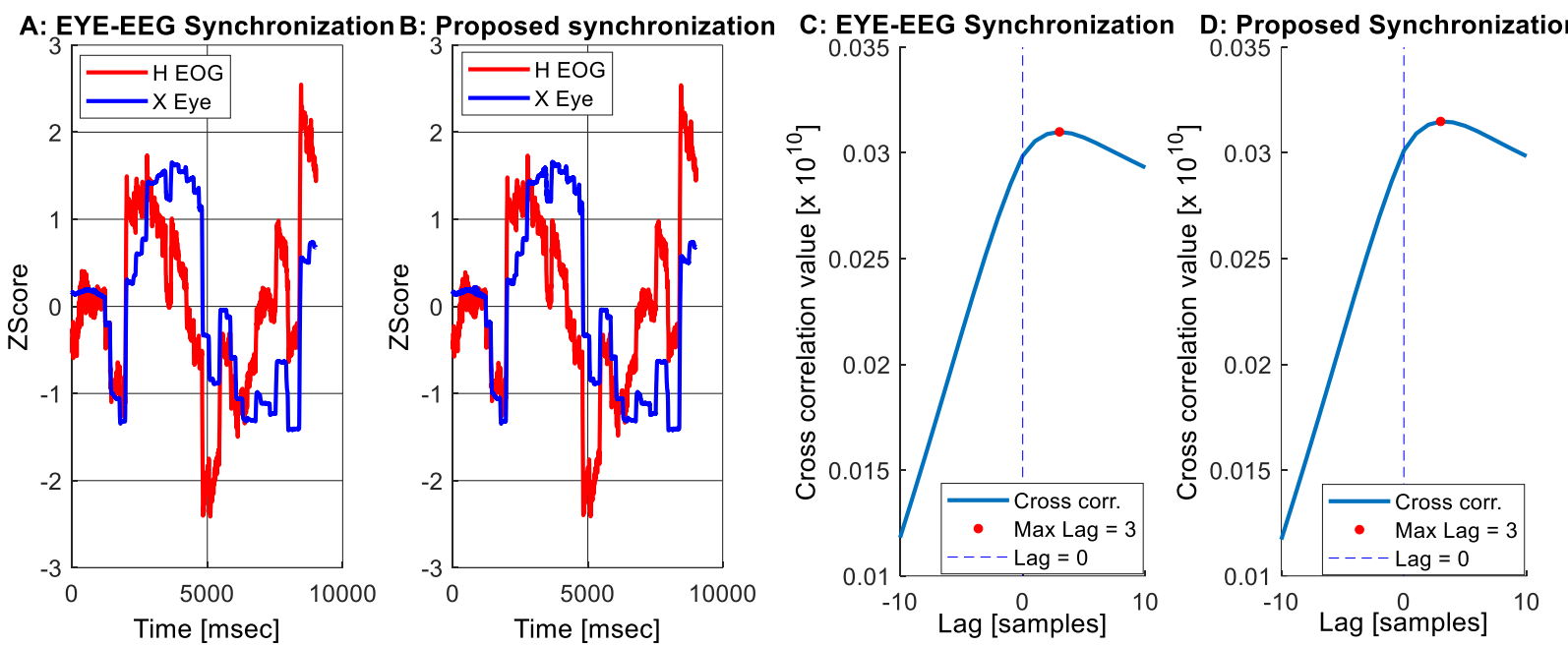

701

702

703

704

705

706

707

708

709

Figure 10: For the scenario 3 dataset, (A, B) evolution of the horizontal eye position and the horizontal EOG (H EOG) signals obtained from a randomly-selected trial, after centering and reduction (ZScore) to enable superposition on the same scale, $(C, D)$ cross correlation between these two signals, for $(A, C)$ the EYE-EEG synchronization and $(\mathrm{B}, \mathrm{D})$ the proposed synchronization

All these features based on alignment errors and cross-correlation were computed on scenario 2 datasets for each method of synchronization. Table 4 shows the statistical summary for each feature and each implementation.

Proposed EYE-EEG Student test

synchronization method

\begin{tabular}{llll}
\hline Mean [samples] & $-0.015(0.062)$ & $-0.020(0.036)$ & $\mathrm{t}(47)=0.030, \mathrm{p}=0.977$ \\
Standard dev. [samples] & $0.551(0.007)$ & $0.699(0.005)$ & $\mathrm{t}(47)=-4.220, \mathbf{p}<\mathbf{0 . 0 0 1} * * *$ \\
Kurtosis & $2.604(0.024)$ & $2.695(0.030)$ & $\mathrm{t}(47)=13.619, \mathbf{p}<\mathbf{0 . 0 0 1} * * *$ \\
Skewness & $0.110(0.116)$ & $-0.064(0.050)$ & $\mathrm{t}(47)=1.393, \mathrm{p}=0.170$ \\
Cross corr. lag [samples] & $-0.875(0.151)$ & $-1.313(0.196)$ & $\mathrm{t}(47)=2.445, \mathbf{p}=\mathbf{0 . 0 1 8 *}$
\end{tabular}

Table 4. Statistical summary. Keys: average (standard error) of the quality features for both implementations (column 2, 3); results of the Student tests (column 4), for four statistical moments (Mean, Standard deviation, Kurtosis and Skewness) and the cross-correlation lag. ${ }^{*} \mathrm{p}<0.5,{ }^{*} * \mathrm{p}<0.001$, bold:

713 significant effect. 
Synchronization of acquisition devices in neuroimaging:

An application using co-registration of eye movements and electroencephalography

714 Results for the spread feature (std) and the cross-correlation lag ${ }^{7}$ were significantly better with our

715 synchronization method, whereas the flatness (Kurtosis) was significantly better with the EYE-EEG

716 synchronization method.

717

$718 \quad$ Processing time

719 On average, our implementation ran 7.5 times faster than the EYE-EEG synchronization for

720 scenario 2 datasets, on the same computer. This result is mainly due to the fact that programing languages

721 are different in both implementations $(\mathrm{C}++$ for ours and Matlab® for EYE-EEG). More interestingly, Table

7225 shows the percentage of time spent on data loading compared to synchronization computation. In our

723 implementation, most of the time was devoted to data loading and implementation of the synchronization

724 algorithm was optimized, making it extremely fast.

725

Eye tracking data [\%]

EEG data [\%]

Synchronization [\%]

Proposed

synchronization method

55.2

44.5

0.3

EYE-EEG

79.2

14.1

6.7

Table 5. Comparison of the percentage of time spent on data loading (eye-tracker data and EEG data) and on synchronization.

7 EOG signals were not recorded for scenario 2 datasets. For cross-correlation estimation, EEG channels closest to the external canthus of the right/left eye were therefore chosen: F8 (right) and F7 (left) channels. 
Synchronization of acquisition devices in neuroimaging:

An application using co-registration of eye movements and electroencephalography

\section{Conclusion}

In this article, we present an original method of data synchronization, generated by acquisition

732 devices working independently. The core of the method rests on a classical synchronization paradigm using

733 external triggers. However, we have shown that this paradigm alone does not provide a sufficiently robust

734 synchronization when dealing with a wide range of problematic situations. We therefore proposed a

735 complete procedure, which we tested and compared with the open source EYE-EEG toolbox on simulated

736 data and on real data from co-registrations of eye movements and EEG.

737 Our procedure specifically aimed to compensate in situations with missing/spurious triggers in the

738 different data flows, and for use of the Pause/Resume mode by the experimenter during acquisition.

739 Common triggers marking the beginning and the end of acquisition must be accurately detected. If

740 synchronization is based only on the detection of these two common triggers, results can be inconsistent

741 when these triggers are missing, for example, if the experimenter starts the acquisition of the secondary

742 device too late or stops it too early. In such situations, the EYE-EEG toolbox requires a manual search in

743 raw files in order to choose other common triggers. Our procedure has the advantage of automatically

744 rectifying errors generated by the experimenter. In addition, if electronic circuits are relied on to sample

745 trigger signals, spurious triggers corresponding to transient states on one sampling period can appear and

746 render the matching process ambiguous. For this reason, we proposed the use of the LCS algorithm for the

747 matching of trigger sequences. We showed that this flexible matching algorithm is a very useful first step

748 before clock drift correction.

749 Moreover, as we have pointed out, there is a growing interest in co-registration experiments using

750 complementary experimental modalities. However, these experiments are often fairly long, and may require

751 pauses, which can either be pre-planned to allow participants to rest for example, or unplanned, when 
Synchronization of acquisition devices in neuroimaging:

An application using co-registration of eye movements and electroencephalography

752 technical problems occur on one or other of the recording devices. In both cases, the use of the

753 "Pause/Resume" mode makes it possible to limit the size of the recorded files, and to facilitate subsequent

754 processing on samples/files that contain only informative data. It is therefore important to have a

755 synchronization procedure which takes these pauses into consideration and manages them correctly. To this

756 end, we implemented a piecewise linear regression between the common triggers expressed in number of

757 samples in each data flow. Drift correction was not, therefore, carried out across all trigger sequences and

758 was instead done locally on each acquisition phase between the successive pauses. Shift values were

759 automatically calculated by the algorithm to compensate for pauses. By executing our procedure on real

760 data corresponding to an acquisition of around one hour, we observed very few samples of residual drift.

761 After synchronization, quality can be assessed from the distribution of alignment errors, and from

762 cross-correlation between horizontal EOG and eye position. The spread of the distribution of alignment

763 errors is on average larger when synchronization is carried out with the EYE-EEG toolbox. However, this

764 distribution spread which is larger than in our method, is compensated for, on average, by a better shape

765 with a sharper distribution with EYE-EEG synchronization. For the cross-correlation, the lags between

766 horizontal EOG and eye positions, are on average longer when synchronization is carried out using the

767 EYE-EEG toolbox. It should be noted that even if the differences between the lags are significant for both

768 methods, they remain small with EYE-EEG synchronization (around one sample on average). However, it

769 is still essential to have the lowest possible number of errors and a small lag in order to limit any potential

770 bias when interpreting the results, especially in experiments using methods aimed at obtaining high-quality

771 temporal resolution. Based on these qualitative criteria, the two synchronization methods are of similar

772 overall quality, ideally when no pauses occur. The EYE-EEG method relies on only one regression,

773 whereas many regressions can be implemented in our method (i.e., the number of regressions is equal to the 
Synchronization of acquisition devices in neuroimaging:

An application using co-registration of eye movements and electroencephalography

774 number of recordings separated by pauses). This limits the use of the EYE-EEG method to experiments 775 without pauses.

776 Finally in keeping with current trends, and with the demands of science, the proposed

777 synchronization algorithm was implemented in $\mathrm{C}++$ language. Consequently, it has a very rapid execution

778 time (about $15 \mathrm{~s}$ for one hour of acquisition). The resulting software in the form of a DOS application has

779 been extensively and successfully tested in a number of situations. The software provided is easy to handle

780 -a tutorial with instructions for users is provided in the "Supplementary Material"- and it does not require

781 any graphical environment. Moreover, it can be easily extended by developing in front-end, data

782 conversion functions which enables it to deal with the increasingly large number of manufacturers.

783 The development of robust procedures of synchronization is very important, because joint analysis

784 of multimodal datasets aims to combine the complementary aspects of each modality in such a way that

785 there is an added benefit compared to analyzing and interpreting each dataset separately. In addition, and in

786 view of the growing number of EFRP experiments, this could create substantial added value for

787 neuroscience applications. Multimodal data integration could lead to a more comprehensive view of brain

788 processes and structures (Uludağ \& Roebroeck, 2014), and the next step could be to develop an online

789 solution with the same qualities of flexibility and robustness in the near future.

790

791

792

793 
Synchronization of acquisition devices in neuroimaging:

An application using co-registration of eye movements and electroencephalography

\section{Acknowledgments}

With a great deal of emotion, we dedicate this article to Gelu Ionescu, research engineer at the 797 GIPSA-Lab, who was at the origin and the development of the vast majority of this work, and who died in 798 a climbing accident a few days after his retirement. May this manuscript be a reflection of our eternal 799 gratitude for his investment in his daily work and his kindness.

\section{Funding}

802 This work was funded in part by a grant from the French National Research Agency (ANR) under 803 the project GAZE\&EEG (ANR-09-BLAN-0330) and a grant from the LabEx PERSYVAL-Lab (ANR-11$804 \quad$ LABX-0025-01)

The executable program and code as well as datasets (coming from eye-tracker and EEG acquisition 807 devices, according to the examples explained in this article) are available in the Zenodo repository:

808 http://doi.org/10.5281/zenodo.4897128

809

\section{References}

811 Altschul, S. F., Gish, W., Miller, W., Myers, E. W., \& Lipman, D. J. (1990). Basic local alignment search 812 tool. Journal of Molecular Biology, 215(3), 403-410. https://doi.org/10.1016/S0022-2836(05)80360-2

813 Bergroth, L., Hakonen, H., \& Raita, T. (2000). A survey of longest common subsequence algorithms. $814 \quad$ Proceedings Seventh International Symposium on String Processing and Information Retrieval, 39- 
Synchronization of acquisition devices in neuroimaging:

An application using co-registration of eye movements and electroencephalography 48.

816 Delorme, A., \& Makeig, S. (2004). EEGLAB: An open source toolbox for analysis of single-trial EEG

817 dynamics including independent component analysis. Journal of Neuroscience Methods, 134(1), 9-21. 818 https://doi.org/10.1016/j.jneumeth.2003.10.009

819 Deriche, R. (1990). Fast Algorithms for Low-Level Vision. IEEE Transactions on Pattern Analysis and $820 \quad$ Machine Intelligence, 12(1), 78-87.

821 Devillez, H., Guyader, N., \& Guérin-Dugué, A. (2015). An eye fixation-related potentials analysis of the 822 P300 potential for fixations onto a target object when exploring natural scenes. Journal of Vision,

824 Dimigen, O., Sommer, W., Hohlfeld, A., Jacobs, A. M., \& Kliegl, R. (2011). Coregistration of eye 825 movements and EEG in natural reading: Analyses and review. Journal of Experimental Psychology: General, 140(4), 552-572. https://doi.org/10.1037/a0023885

Ehinger, B. V., \& Dimigen, O. (2019). Unfold: An integrated toolbox for overlap correction, non-linear modeling, and regression-based EEG analysis. PeerJ, 2019(10), 0-33. https://doi.org/10.7717/peerj.7838

830 Engbert, R., \& Kliegl, R. (2003). Microsaccades uncover the orientation of covert attention. Vision Research, 43(9), 1035-1045. https://doi.org/10.1016/S0042-6989(03)00084-1

832 Frey, A., Ionescu, G., Lemaire, B., López-Orozco, F., Baccino, T., \& Guérin-Dugué, A. (2013). Decision833 making in information seeking on texts: An Eye-Fixation-Related Potentials investigation. Frontiers 834 in Systems Neuroscience, JUL. https://doi.org/10.3389/fnsys.2013.00039

835 Frey, A., Lemaire, B., Vercueil, L., \& Guérin-Dugué, A. (2018). An Eye Fixation-Related Potential Study 836 in Two Reading Tasks: Reading to Memorize and Reading to Make a Decision. Brain Topography, 837 31(4), 640-660. https://doi.org/10.1007/s10548-018-0629-8 
Synchronization of acquisition devices in neuroimaging:

An application using co-registration of eye movements and electroencephalography

838 Guérin-Dugué, A., Roy, R. N., Kristensen, E., Rivet, B., Vercueil, L., \& Tcherkassof, A. (2018). Temporal

839 dynamics of natural static emotional facial expressions decoding: A study using event- and eye

840 fixation-related potentials. Frontiers in Psychology, 9(JUN), 1-19.

$841 \quad$ https://doi.org/10.3389/fpsyg.2018.01190

842 Hoogeboom, P. J. (2003). Off-line synchronization of measurements based on a common pseudorandom

843 binary signal. Behavior Research Methods, Instruments, \& Computers, 35(3), 384-390.

844 Jorge, J., Grouiller, F., Ipek, Ö., Stoermer, R., Michel, C. M., Figueiredo, P., van der Zwaag, W., \&

845 Gruetter, R. (2015). Simultaneous EEG-fMRI at ultra-high field: artifact prevention and safety

$846 \quad$ assessment. Neuroimage, 105, 132-144.

847 Jung, T. P., Makeig, S., Westerfield, M., Townsend, J., Courchesne, E., \& Sejnowski, T. J. (2000).

848 Removal of eye activity artifacts from visual event-related potentials in normal and clinical subjects.

849 Clinical Neurophysiology, 111(10), 1745-1758. https://doi.org/10.1016/S1388-2457(00)00386-2

850 Kamienkowski, J. E., Ison, M. J., Quiroga, R. Q., \& Sigman, M. (2012). Fixation-related potentials in

851 visual search: A combined EEG and eye tracking study. Journal of Vision, 12(7), 1-20.

$852 \quad$ https://doi.org/10.1167/12.7.4

853 Körner, C., Braunstein, V., Stangl, M., Schlögl, A., Neuper, C., \& Ischebeck, A. (2014). Sequential effects

854 in continued visual search: Using fixation-related potentials to compare distractor processing before

855 and after target detection. Psychophysiology, 51(4), 385-395. https://doi.org/10.1111/psyp.12062

856 Kristensen, E., Guerin-Dugué, A., \& Rivet, B. (2017). Regularization and a general linear model for event-

857 related potential estimation. Behavior Research Methods, 49(6), 2255-2274.

$858 \quad$ https://doi.org/10.3758/s13428-017-0856-z

859 Kristensen, E., Rivet, B., \& Guerin-Dugué, A. (2017). Estimation of overlapped Eye Fixation Related

860 Potentials: The General Linear Model, a more flexible framework than the ADJAR algorithm. Journal 
Synchronization of acquisition devices in neuroimaging:

An application using co-registration of eye movements and electroencephalography of Eye Movement Research, 10(1), 1-27. https://doi.org/10.16910/jemr.10.1.7

862 Liu, Z., Ding, L., \& He, B. (2006). Integration of EEG/MEG with MRI and fMRI in Functional

863 Neuroimaging. IEEE Engineering in Medicine and Biology Magazine: The Quarterly Magazine of the 864 Engineering in Medicine \& Biology Society, 25(4), 46-53.

865 Nikolaev, A. R., Meghanathan, R. N., \& van Leeuwen, C. (2016). Combining EEG and eye movement 866 recording in free viewing: Pitfalls and possibilities. Brain and Cognition, 107, 55-83.

867 https://doi.org/10.1016/j.bandc.2016.06.004

868 Nikolaev, A. R., Pannasch, S., Ito, J., \& Belopolsky, A. V. (2014). Eye movement-related brain activity 869 during perceptual and cognitive processing. Frontiers in Systems Neuroscience, 8(1 APR), 2013870 2014. https://doi.org/10.3389/fnsys.2014.00062

871 Pak, W. (2017). Ultra-low-power media access control protocol based on clock drift characteristics in 872 wireless sensor networks. International Journal of Distributed Sensor Networks, 13(7). https://doi.org/10.1177/1550147717722155

874 Picton, T. W., Bentin, S., Berg, P., Donchin, E., Hillyard, S. A., Johnson, R., Miller, G. A., Ritter, W., 875 Ruchkin, D. S., Rugg, M. D., \& Taylor, M. J. (2000). Guidelines for using human event-related 876 potentials to study cognition: Recording standards and publication criteria. Psychophysiology, 37(2), 127-152. https://doi.org/10.1017/S0048577200000305

878 Rosenkranz, K., \& Lemieux, L. (2010). Present and future of simultaneous EEG-fMRI. Magnetic 879 Resonance Materials in Physics, Biology and Medicine, 23(5), 309-316.

880 Shin, J., von Lühmann, A., Kim, D. W., Mehnert, J., Hwang, H. J., \& Müller, K. R. (2018). Simultaneous 881 acquisition of EEG and NIRS during cognitive tasks for an open access dataset. Scientific Data, $8825(180003), 1-16$.

883 Uludağ, K., \& Roebroeck, A. (2014). General overview on the merits of multimodal neuroimaging data 
Synchronization of acquisition devices in neuroimaging:

An application using co-registration of eye movements and electroencephalography fusion. NeuroImage, 102(1), 3-10.

885 Van Humbeeck, N., Meghanathan, R. N., Wagemans, J., Leeuwen, C., \& Nikolaev, A. (2018). Presaccadic

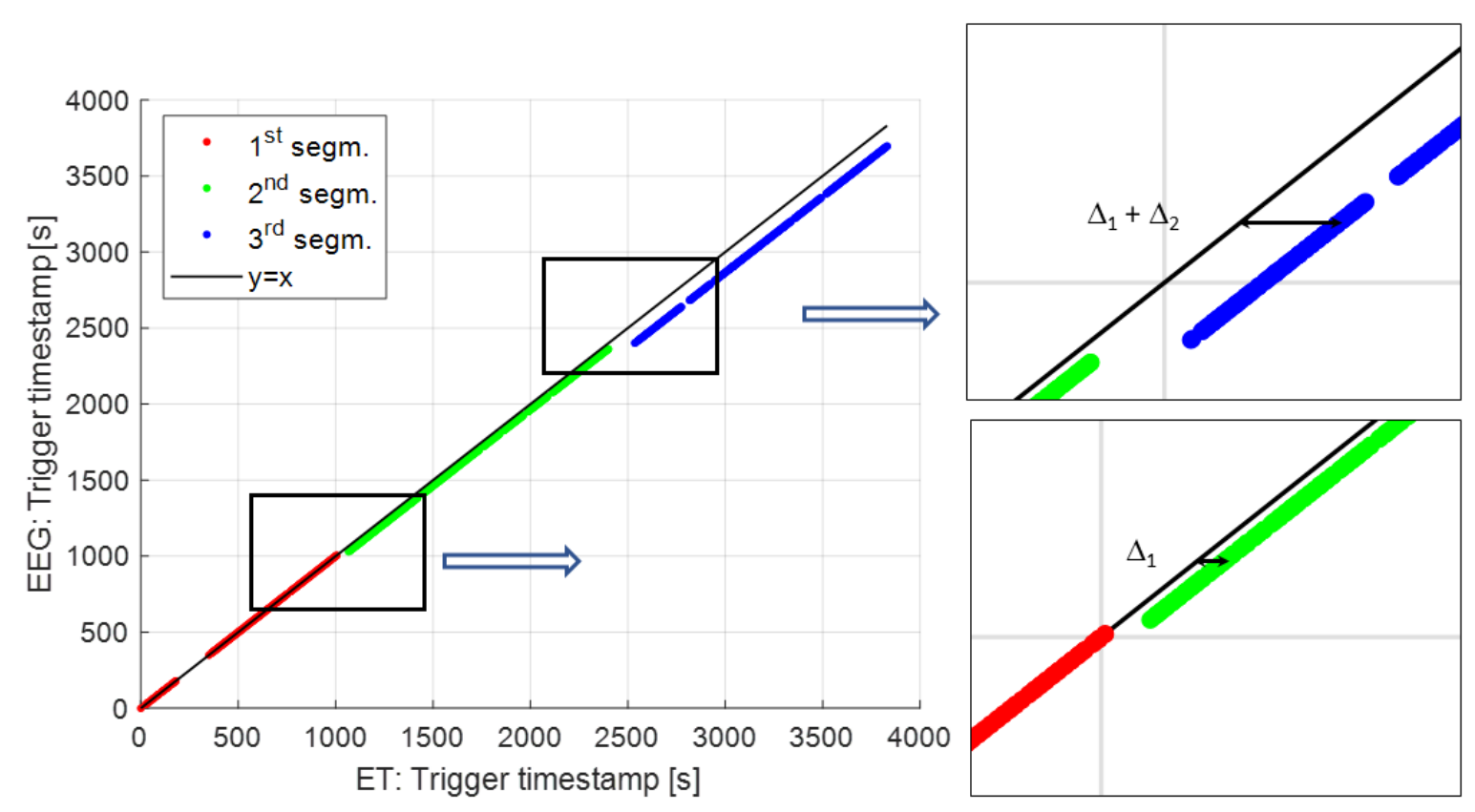

894 Figure A1. Joint representation of the timestamps of each common trigger in both modalities before 895 synchronization. $\Delta 1$ and $\Delta 2$ are the observed time shifts that must be corrected by the drift and shift 896 corrections. 
Synchronization of acquisition devices in neuroimaging:

An application using co-registration of eye movements and electroencephalography

900 the gap between their timestamps in each modality was larger than the tolerance parameter (by default, 4

901 samples). Only one regression was performed and the additional shifts resulting from the two pauses could

902 not be compensated. This synchronization default was confirmed by the cross correlation between the

903 horizontal eye position and the differential EEG signal computed from the F8 channel and the F7 channel

904 (Figure A2 B). The histogram of alignment errors, using the proposed method on the 1222 common

905 triggers, is shown in Figure 6 D. The additional shifts were found by performing the piecewise linear

906 regression (shift correction). The cross correlation is shown in Figure A2 C.

907
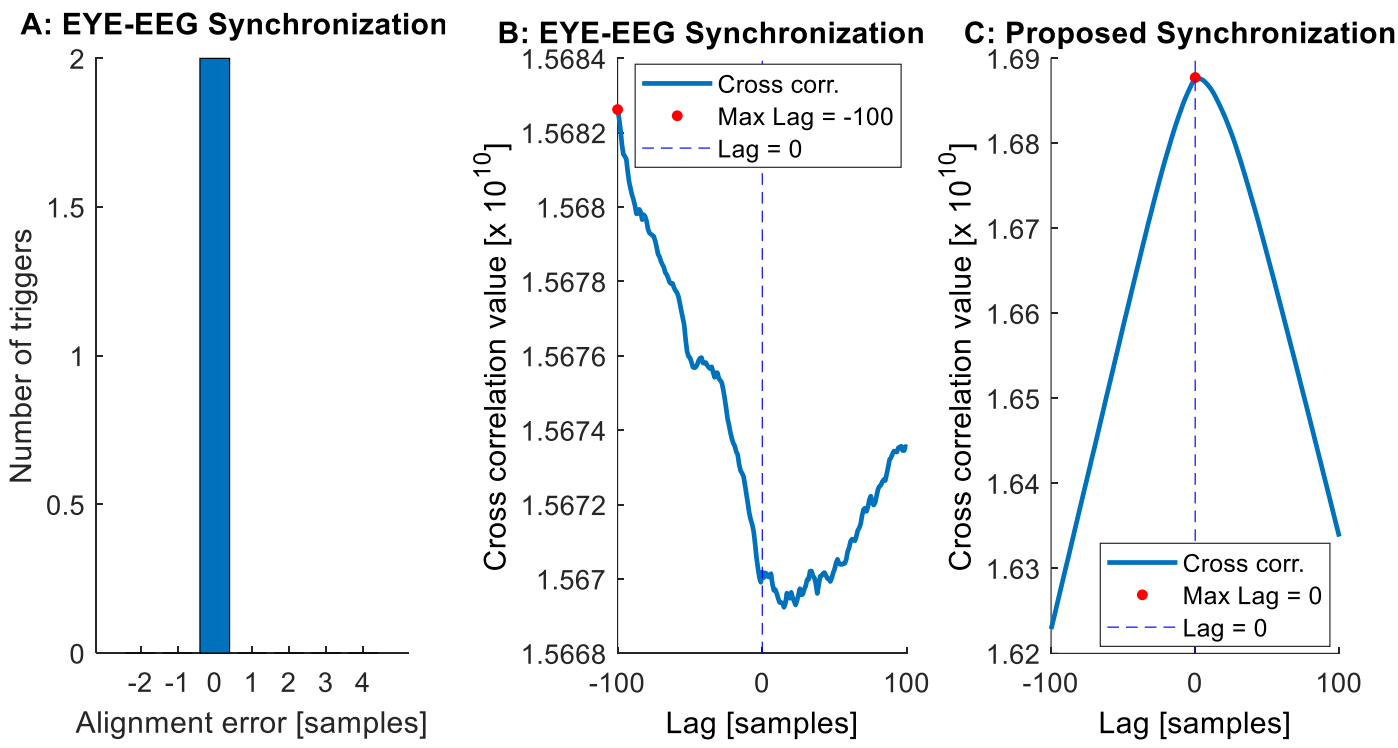

Figure A2. Synchronization of a scenario 1.3 dataset containing two pauses. (A) Histogram of alignment errors on the common triggers found by the EYE-EEG method. (B) Cross correlation between the horizontal eye position and the F8-F7 EEG signals, for the EYE-EEG synchronization and (C) for the 